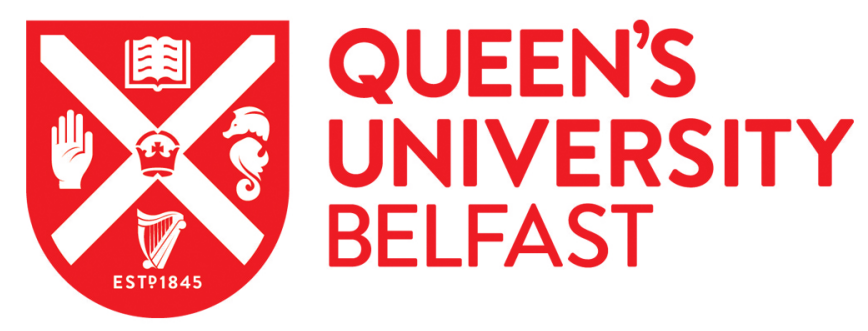

\title{
ELOVL5 is a critical and targetable fatty acid elongase in prostate cancer
}

Centenera, M. M., Scott, J. S., Machiels, J., Nassar, Z. D., Miller, D. C., Zininos, I., Dehairs, J., Burvenich, I. J. G., Zadra, G., Chetta, P., Bango, C., Evergren, E., Ryan, N. K., Gillis, J. L., Mah, C. Y., Tieu, T., Hanson, A. R., Carelli, R., Bloch, K., ... Butler, L. M. (2021). ELOVL5 is a critical and targetable fatty acid elongase in prostate cancer. Cancer Research. https://doi.org/10.1158/0008-5472.CAN-20-2511

\section{Published in: \\ Cancer Research}

\section{Document Version:}

Peer reviewed version

\section{Queen's University Belfast - Research Portal:}

Link to publication record in Queen's University Belfast Research Portal

\section{Publisher rights}

Copyright 2021 American Association for Cancer Research. This work is made available online in accordance with the publisher's policies. Please refer to any applicable terms of use of the publisher.

\section{General rights}

Copyright for the publications made accessible via the Queen's University Belfast Research Portal is retained by the author(s) and / or other copyright owners and it is a condition of accessing these publications that users recognise and abide by the legal requirements associated with these rights.

Take down policy

The Research Portal is Queen's institutional repository that provides access to Queen's research output. Every effort has been made to ensure that content in the Research Portal does not infringe any person's rights, or applicable UK laws. If you discover content in the Research Portal that you believe breaches copyright or violates any law, please contact openaccess@qub.ac.uk. 


\section{ELOVL5 is a critical and targetable fatty acid elongase in prostate cancer}

Margaret M. Centenera ${ }^{1,2,3}$, Julia S. Scott ${ }^{1,2,3}$, Jelle Machiels ${ }^{4}$, Zeyad D. Nassar ${ }^{1,2,3}$,

Deanna C. Miller ${ }^{1,2,3}$, Irene Zinonos ${ }^{1,5}$, Jonas Dehairs ${ }^{4}$, Ingrid J. G. Burvenich ${ }^{6,7}$, Giorgia Zadra $^{8}$, Paolo M. Chetta ${ }^{8,9, \#}$, Clyde Bango ${ }^{8}$, Emma Evergren $^{10}$, Natalie K. Ryan ${ }^{1,3}$, Joanna L. Gillis ${ }^{1,3}$, Chui Yan Mah ${ }^{1,2,3}$, Terence Tieu ${ }^{11,12}$, Adrienne R. Hanson ${ }^{1}$, Ryan Carelli ${ }^{13}$, Katarzyna Bloch ${ }^{4}$, Vasilios Panagopoulos ${ }^{1,3}$, Etienne Waelkens ${ }^{14}$, Rita Derua ${ }^{14}$, Elizabeth D. Williams ${ }^{15}$, Andreas Evdokiou ${ }^{1,5}$, Anna Cifuentes-Rius ${ }^{11}$, Nicolas H. Voelcker ${ }^{11,12,16}$, lan G. Mills ${ }^{10,17}$, Wayne D. Tilley ${ }^{1,2}$, Andrew M. Scott $6,7,18,19$, Massimo Loda ${ }^{13}$, Luke A. Selth $^{1,2,20}$, Johannes V. Swinnen ${ }^{4,{ }^{*}}$ and Lisa M. Butler ${ }^{1,2,3,{ }^{*}}$

${ }^{1}$ University of Adelaide Medical School, Adelaide, SA, Australia

${ }^{2}$ Freemasons Foundation Centre for Men's Health, Adelaide, SA, Australia

${ }^{3}$ South Australian Health and Medical Research Institute, Adelaide, SA, Australia

${ }^{4}$ Department of Oncology, Laboratory of Lipid Metabolism and Cancer, KU Leuven, Leuven, Belgium

${ }^{5}$ Basil Hetzel Institute, Queen Elizabeth Hospital, SA, Australia

${ }^{6}$ Tumour Targeting Laboratory, Olivia Newton-John Cancer Research Institute, Melbourne, VIC, Australia

${ }^{7}$ School of Cancer Medicine, La Trobe University, Melbourne, VIC, Australia

${ }^{8}$ Dana-Farber Cancer Institute, Boston, MA, USA

${ }^{9}$ University of Milan, Milan, Italy

${ }^{10}$ Patrick G Johnston Centre for Cancer Research, Queen's University Belfast, Belfast, UK

${ }^{11}$ Drug Delivery, Disposition and Dynamics, Monash University, Parkville, VIC, Australia 
${ }^{12}$ Commonwealth Scientific and Industrial Research Organisation (CSIRO), Clayton, VIC, Australia.

${ }^{13}$ Department of Pathology and Laboratory Medicine, Weill Cornell Medicine, New YorkPresbyterian Hospital, New York, NY, USA

${ }^{14}$ Department of Cellular and Molecular Medicine, KU Leuven, Leuven, Belgium

${ }^{15}$ Australian Prostate Cancer Research Centre - Queensland, Queensland University of Technology (QUT), Princess Alexandra Hospital, Translational Research Institute, Brisbane, QLD, Australia

${ }^{16}$ Melbourne Centre for Nanofabrication, Victorian Node of the Australian National Fabrication Facility, Clayton, VIC, Australia

${ }^{17}$ Nuffield Department of Surgical Sciences, University of Oxford, Oxford UK

${ }^{18}$ Department of Medicine, University of Melbourne, Melbourne, VIC, Australia

${ }^{19}$ Department of Molecular Imaging and Therapy, Austin Health, Melbourne, VIC, Australia

${ }^{20}$ Flinders Health and Medical Research Institute, Flinders University, Bedford Park, South Australia, 5042 Australia

${ }^{\#}$ Current employee of Boehringer-Ingelheim RCV GmbH \& Co KG, Vienna, Austria ${ }^{*}$ LM Butler and JV Swinnen jointly supervised this work

Running title: Targeting ELOVL5 in prostate cancer

Key words: lipid metabolism, prostate cancer, phospholipid elongation, androgen receptor, ELOVL5, lipidomics, patient-derived explants

Corresponding Authors: Prof Lisa Butler, Adelaide Medical School, University of Adelaide, c/o Cancer Theme, Level 5N, SAHMRI, PO Box 11060, Adelaide, SA 5001, Australia. Phone: (+61) 88128 4360; Email: lisa.butler@adelaide.edu.au; Prof Johan 
Swinnen, Laboratory of Lipid Metabolism and Cancer, KU Leuven, O\&N I Herestraat 49 box 818, Leuven 3000, Belgium. Phone: +32 163305 33; Email: j.swinnen@kuleuven.be Conflict of interest disclosure statement: The authors declare no financial and/or nonfinancial competing interests

Word Count: 6,082

Figures \& Tables: 7 figures 


\section{Abstract}

The androgen receptor (AR) is the key oncogenic driver of prostate cancer, and despite implementation of novel AR targeting therapies, outcomes for metastatic disease remain dismal. There is an urgent need to better understand androgen-regulated cellular processes to more effectively target the AR-dependence of prostate cancer cells through new therapeutic vulnerabilities. Transcriptomic studies have consistently identified lipid metabolism as a hallmark of enhanced AR signaling in prostate cancer, yet the relationship between $A R$ and the lipidome remain undefined. Using mass spectrometrybased lipidomics, this study reveals increased fatty acyl chain length in phospholipids from prostate cancer cells and patient-derived explants as one of the most striking androgenregulated changes to lipid metabolism. Potent and direct AR-mediated induction of ELOVL fatty acid elongase 5 (ELOVL5), an enzyme that catalyzes fatty acid elongation, was demonstrated in prostate cancer cells, xenografts, and clinical tumors. Assessment of mRNA and protein in large-scale datasets revealed ELOVL5 as the predominant ELOVL expressed and upregulated in prostate cancer compared to non-malignant prostate. ELOVL5 depletion markedly altered mitochondrial morphology and function, leading to excess generation of reactive oxygen species and resulting in suppression of prostate cancer cell proliferation, 3D growth, and in vivo tumor growth and metastasis. Supplementation with the monounsaturated fatty acid cis-vaccenic acid, a direct product of ELOVL5 elongation, reversed the oxidative stress and associated cell proliferation and migration effects of ELOVL5 knockdown. Collectively, these results identify lipid elongation as a pro-tumorigenic metabolic pathway in prostate cancer that is androgen-regulated, critical for metastasis, and targetable via ELOVL5. 


\section{Significance Statement}

This study identifies phospholipid elongation as a new metabolic target of androgen action that is critical for prostate tumor metastasis. 


\section{Introduction}

Prostate cancer is the most common cancer diagnosed in men from the developed world, and is a major cause of mortality (1). Androgens acting through the androgen receptor (AR) transcription factor drive prostate cancer initiation and progression. Accordingly, androgen deprivation therapy (ADT) has remained the frontline treatment strategy for advanced prostate cancer since the 1940s. Although initially effective, ADT fails to achieve an enduring remission and patients ultimately develop castration resistant prostate cancer (CRPC), which has a poor prognosis despite the clinical implementation of new ARdirected agents (2). Underpinning the development of more effective therapeutic strategies is a more detailed understanding of the critical cellular processes through which androgens regulate prostate tumor biology and promote cancer cell survival.

It is well established that androgens have a major stimulatory effect on de novo lipogenesis in prostate cancer cells (3). This synthesis of fatty acids is activated in the majority of prostate cancers, and is associated with poor prognosis $(4,5)$. Approximately $80 \%$ of de novo synthesized fatty acids are utilized for the production of phospholipids, the dominant component of cellular membranes, and is linked to the tumor cell's increased demand for cellular membranes and their protection from oxidative stress (6). This biology has elicited major efforts to target lipid synthesis therapeutically via inhibition of the key lipogenesis enzyme, fatty acid synthase $(F A S M)$. FASN is directly and indirectly regulated by the AR and although FASN inhibitors have proved promising in the preclinical setting, off-target effects, poor solubility and pharmacokinetics have prevented their clinical development (7).

It is known that androgens stimulate expression of over 20 enzymes involved in lipid synthesis, binding, uptake, metabolism and transport $(8,9)$, however the precise regulatory mechanisms and influence of these factors on lipid composition and metabolism in 
prostate tumor cells remain largely undefined. The aim of this study was to gain insight into regulation of the prostate tumor lipidome by androgens in prostate cancer cells, and ultimately identify novel metabolic effectors of androgen action for therapeutic intervention. 


\section{Materials and Methods}

\section{Cell culture and treatments}

Human prostate cancer cell lines LNCaP (clone FGC), 22Rv1 and LAPC4 were from the American Type Culture Collection (Manassas, USA). All cell lines were authenticated by short tandem repeat profiling at Cell Bank Australia (NSW, Australia) in July 2016 and again in July 2020. Cell lines were cultured in RPMI-1640 medium (Life Technologies, Carlsbad, USA) containing 10\% fetal bovine serum (FBS) and 2mM L-Glutamine (Life Technologies). For androgen treatment, cells were initially seeded in medium containing $5 \%$ dextran charcoal coated FBS (dcc-FBS) and after $24 \mathrm{~h}, 1 \mathrm{nM}$ mibolerone or DHT added and cells cultured for an additional $72 \mathrm{~h}$. For anti-androgen treatment, LNCaP cells were cultured in growth medium containing $1 \mu \mathrm{M}$ enzalutamide for $72 \mathrm{~h}$.

\section{Patient-derived explant (PDE) tissue culture}

PDE experiments were conducted according to Declaration of Helsinki principles. Prostate tumor specimens were obtained with written informed consent through the Australian Prostate Cancer Bioresource from men undergoing robotic radical prostatectomy at St Andrew's Hospital (Adelaide, Australia). Tumors were cultured as PDEs for $48 \mathrm{~h}$ in the presence or absence of $10 \mu \mathrm{M}$ enzalutamide, as previously described (10). Half was formalin-fixed and paraffin embedded for histology and half was snap frozen for lipidomics.

\section{Electrospray ionization tandem mass spectrometry (ESI-MS/MS)-based lipidomics}

Cells or tissues were homogenized in ice cold $800 \mu$ phosphate buffered saline (PBS). $100 \mu \mathrm{l}$ cell/explant lysate was set aside to quantify DNA for normalization then lipids extracted using a modified Bligh-Dyer protocol by adding $800 \mu \mathrm{CHCl}_{3}, 900 \mu \mathrm{l} 1 \mathrm{~N}$ $\mathrm{HCl}: \mathrm{CH}_{3} \mathrm{OH}$ 1:8 (v/v) and $500 \mu \mathrm{g}$ of the anti-oxidant 2,6-di-tert-butyl-4-methylphenol (Sigma). Following centrifugal phase separation, the lower organic fraction was collected, evaporated, and the lipid pellet stored under argon gas at $-20^{\circ} \mathrm{C}$. Lipid pellets were 
reconstituted in diluent $\left(\mathrm{CH}_{3} \mathrm{OH}: \mathrm{CHCl}_{3}: \mathrm{NH}_{4} \mathrm{OH} ; 90: 10: 1.25, \mathrm{v} / \mathrm{v} / \mathrm{v}\right)$ according to DNA quantification $(1 \mu$ diluent / $1 \mu \mathrm{g}$ DNA). Phospholipids were analyzed by ESI-MS/MS on a hybrid quadrupole linear ion trap mass spectrometer (4000 QTRAP system, AB SCIEX) equipped with a TriVersa NanoMate robotic nanosource (Advion Biosciences) as described previously (6).

\section{Quantitative Real Time PCR}

RNA was extracted using the PureLink TM Total RNA purification system (Life Technologies) according to the manufacturer's instructions, and concentration assessed using a NanoDrop DM-1000 spectrophotometer (NanoDrop Technologies). RNA was reverse transcribed using Superscript II RT and random hexamer primers according to the manufacturer's instructions (Invitrogen). RT-qPCR was performed on a 7500 Fast RealTime system (Applied Biosystems) using primer sequences detailed in Supplementary Table 2. Expression levels were normalized against 18S, GUSB or L19 as reference genes.

\section{Gene Set Enrichment Analysis (GSEA)}

GSEA was implemented using the Broad Institute's public GenePattern server essentially as described previously (11). Briefly, the correlation between expression levels of ELOVL2, ELOVL5, ELOVL7 and 26,447 genes was calculated using data from a cohort of 131 patients from MSKCC (12). Genes were subsequently ranked according to Pearson correlation coefficient ( $r$ ) value, and this ranked gene list was used for GSEA Pre-ranked analysis.

\section{Analysis of published ChIP-seq data}

AR ChIP-seq data from clinical specimens (7 normal prostate and 13 primary tumors) (13) was obtained from GEO (GSE56288). Mean signals from normal and tumor samples were generated using Integrated Genome Browser (14). 


\section{Chromatin immunoprecipitation}

LNCaP cells were seeded at $3 \times 10^{6}$ cells/plate in $15 \mathrm{~cm}$ plates in RPMI- 1640 containing $10 \%$ dcc-FBS for 3 days, then treated for $4 \mathrm{~h}$ with $10 \mathrm{nM}$ DHT or vehicle (ethanol). AR ChIP was performed as described previously (15).

\section{Transient RNA interference}

ON-TARGET plus human small interfering RNAs (siRNAs) and a control siRNA (D001810-01-20 ON-TARGET plus Non-Targeting siRNA \#1) were from Dharmacon. The most effective siRNA from a pool of 4 was selected for targeting ELOVL2 (J-009531-10), ELOVL5 (J-009260-1), ELOVL7 (J-028493-11) and SREBP1 (J-006891-07) and reverse transfected at a concentration of $5 \mathrm{nM}$ using Lipofectamine RNAiMAX transfection reagent (Invitrogen) according to the manufacturer's instructions.

\section{Fluorescence immunohistochemistry of human prostate cancer tissue microarray}

Five tissue microarrays (TMAs) comprised of 1 -mm triplicate tumor cores and single/ duplicate benign tissue cores were used for evaluation of ELOVL5 expression. TMAs were constructed from a cohort of 249 radical prostatectomy prostate cancer patients who had archival tissue available through the Gelb Center at Dana-Farber Cancer Institute (DFCl, Boston, USA). Human studies were conducted according to Declaration of Helsinki principles under institution review board (IRB). A written informed consent was received from participants prior to inclusion in the study.

A multiplex tyramine signal amplification (TSA) method was performed on $4 \mu \mathrm{m}$ TMA sections for detection of ELOVL5 protein, with pan-Cytokeratin and p63 for masking the tumor and benign epithelium, respectively. Staining was performed on a Leica Bond Rx automated immunostainer, using a protocol for sequential TSA-amplified immunofluorescence labels, with DAPI as the nuclear counterstain. Slides were incubated for 30 min with antibodies against ELOVL5 (HPA047752, Sigma, 1:2000), pan-Cytokeratin 
CAM5.2 (452M, Cell Marque, 1:500) and a HMWCK-P63 cocktail comprised of p63 (4892, Cell Signaling Technology, 1:100) and cytokeratin 5/4 (API3025AA, Biocare, ready-touse), followed by 15 min incubation with TSA conjugated Opal dye (Opal 690 for ELOVL5, Opal 520 for pan-Cytokeratin, Opal 570 for HMWCK and p63). A tissue and cell segmentation algorithm was developed in PerkinElmer InForm software and applied to the TMAs. All images were reviewed by a pathologist and manually edited for accuracy before the final segmentation data were generated. Cores with major folding or staining artefacts were excluded. Signal was expressed as fluorescent count normalized to exposure time. Per-cell ELOVL5 cytoplasmic signal was normalized to nuclear DAPI signal before comparison between tumor and benign glands. Unpaired t-test was used when variances were equal, otherwise, non-parametric Mann-Whitney was applied.

\section{Dendrimer functionalized porous silicon nanoparticle (pSiNP) knockdown of ELOVL5}

Alexa Fluor-488 labelled PAMAM(G4)-pSiNPs were prepared as described previously (16), and loaded with either siCTRL or siELOVL5 overnight prior to experimental day. On the day of PDE treatment, loaded NPs were washed $2 \times$ in RNase-free water and added to explant culture media at a concentration of $200 \mu \mathrm{g} / \mathrm{mL}$. Explants were prepared in triplicate as described above and cultured in a 24-well plate containing $500 \mu \mathrm{L}$ NPsiCTRL or NPsiELOVL5 containing growth medium or growth medium without any NPs as a control. After $24 \mathrm{~h}$, an additional $50 \mu \mathrm{L}$ of NP containing media was added and explants cultured for an additional $48 \mathrm{~h}$, making a total culture time of $72 \mathrm{~h}$. At the end of the experiment, explants were formalin-fixed and paraffin embedded prior to immunostaining with antibodies for ELOVL5 (HPA047752, Sigma, 1: 2,000) or Ki67 (M7240, DAKO, 1:200), as described previously (10). Slides were scanned with an Axio Z.1 Scanner (Carl Zeiss Pty. Ltd., NSW, Australia) using 40x objective, and images captured using Zen Blue 2.6 Software (Carl Zeiss Pty. Ltd., NSW, Australia). Ki67 positivity was manually counted, as 
described previously (10). ELOVL5 staining intensity was measured using ImageJ software.

\section{Generation of shELOVL5 LNCaP cells}

LNCaP cells were transduced with universal negative control shRNA lentivirus (pLenti-H1shRNA(Neg-Control)-Rsv(Puro)) or ELOVL5 shRNA lentivirus (pLenti-H1shRNA(hELOVL5)-Rsv(Puro)) designed by GenTarget Inc. (San Diego, CA, USA), according to the manufacturer's instructions. Briefly, $1 \times 10^{5}$ cells were seeded in 24-well plates overnight, then treated with Hexadimethrine Bromide $(8 \mu \mathrm{g} / \mathrm{mL})$ and transduced with shRNA at MOI of 5 . After 3 days, cell selection was undertaken using $4 \mu \mathrm{g} / \mathrm{mL}$ puromycin dihydrochloride (Gibco). ELOVL5 downregulation was confirmed by western blot as described previously (17).

\section{Cell viability assay}

LNCaP or 22Rv1 cells were transfected with siRNA overnight in 24-well plates at a density of $2 \times 10^{4}$ cells/well, then treated as indicated for 6 days and counted as previously described (17). Fatty acids, arachidonic acid (AA), docosahexaenoic acid (DHA) or cisvaccenic acid (cVA) were supplemented as indicated for rescue studies.

\section{$\underline{\text { 3-D spheroid growth assay }}$}

LNCaP cells transfected with siRNA in 6-well plates were collected after $48 \mathrm{~h}$ and suspended at $7.5 \times 10^{4}$ cells $/ \mathrm{mL}$ in RPMI-1640 containing 10\% FBS. Cell suspensions (1500 cells in $20 \mu \mathrm{L}$ ) were pipetted inside the lid of a petri dish and $10-15 \mathrm{~mL}$ PBS added to the base. Dishes were reassembled and incubated at $37^{\circ} \mathrm{C}$. After 5 days, the formed spheres were imaged and sphere volume determined using the equation $V=(L \times W 2) / 2$.

$\underline{\text { Transwell migration assay }}$ 
LNCaP/22Rv1 cells transfected with siRNA were seeded into the upper chamber of 24well polycarbonate Transwell $\circledast$ inserts (3422, Sigma Aldrich, NSW, Australia) at a density of $3.5 \times 10^{4}$ cells/well in RMPI-1640 containing $1 \%$ bovine serum albumin. The bottom chamber was filled with $650 \mu \mathrm{l}$ of RPMI-1640 containing 5\% FBS and cells incubated at 37 ${ }^{\circ} \mathrm{C}$ for $48 \mathrm{~h}$. Transwell membranes were then collected, washed with PBS and nonmigrated cells were scraped from the upper face. The membranes were fixed in $4 \%$ paraformaldehyde for $30 \mathrm{~min}$, stained with hematoxylin overnight and mounted using permount mounting medium. The number of migrated cells was counted microscopically. Results are reported as the number of migrated cells normalized to the number of viable cells counted in a viability assay at the same time point.

\section{Electron Microscopy}

LNCaP cells transfected with siRNA were fixed after $72 \mathrm{~h}$ in $1.25 \%$ glutaraldehyde, $4 \%$ sucrose, $4 \%$ paraformaldehyde in PBS $(\mathrm{pH} 7.2)$ for $1 \mathrm{~h}$ at room temperature, post-fixed in $1 \%$ osmium tetraoxide in water, dehydrated in a graded ethanol series and embedded in epoxy resin Embed812 (Electron Microscopy Science). Ultrathin sections $(70 \mathrm{~nm})$ were cut on a Leica UCT6 ultramicrotome, collected on formvar-coated copper-iridium slot grids, counterstained with $2 \%$ uranylacetate and Reynold's lead citrate, and imaged in a FEI Tecnai G2 Spirit transmission electron microscope at 80kV.

\section{Cellular ROS measurement}

LNCaP cells were transfected with siRNA for 6 days in 24 well plates. For negative controls, cells were treated with $500 \mu \mathrm{M}$ Trolox (Sapphire Bioscience, NSW, Australia) 24 hours prior to collection, followed by treatment with $200 \mu \mathrm{M}$ tert-butyl hydroperoxide (TBHP) (Thermo Fisher Scientific, VIC, Australia) for 1 hour prior to collection. For positive controls, cells were treated with $200 \mu \mathrm{M}$ TBHP for 1 hour prior to collection. Cells were collected into fluorescence-activated sorting (FACS) tubes and stained with $500 \mathrm{nM}$ 
CellROX Orange reagent (Thermo Fisher Scientific, VIC, Australia) for $1 \mathrm{~h}$ at $37^{\circ} \mathrm{C}$. Cells were centrifuged, washed with PBS and then stained with $1 \mu \mathrm{M}$ CellROX Sytox Red Dead reagent (Thermo Fisher Scientific, VIC, Australia) for $15 \mathrm{~min}$ on ice. The samples were then read on a BD LSR Fortessa X20 TM flow cytometer.

\section{Colony formation assay}

ELOVL5 stable downregulated cells (shELOVL5) or negative control cells (shCTRL) plated in 6-well plates (500 cells/well) were incubated for 3 weeks at $37^{\circ} \mathrm{C}$, with medium replenished every 4-7 days. After 3 weeks, cells were washed with PBS, fixed with 4\% paraformaldehyde and stained with $0.5 \%$ crystal violet for $30 \mathrm{~min}$. Colonies with $>50$ cells were counted manually in a blinded manner.

\section{Subcutaneous LNCaP xenograft model}

Subcutaneous xenograft studies were approved by the Austin Health Animal Ethics Committee (approval number A2015/05311). LNCaP cells $\left(5 \times 10^{6}\right.$ cells in $50 \mu \mathrm{L}$ RPMI1640 containing $10 \%$ FBS) were co-injected subcutaneously with $50 \mu \mathrm{L}$ matrigel in 5 to 6 week-old NOD/SCID Gamma male mice (Bioresource Facility, Austin Health, Heidelberg, Australia). When tumors reached $\sim 200 \mathrm{~mm}^{3}$, mice were randomized to three treatment groups; untreated $(n=5)$, vehicle control $(10 \%$ DMSO/PBS; $n=5)$ and enzalutamide (10 $\mathrm{mg} / \mathrm{kg}$ enzalutamide in $10 \%$ DMSO/PBS, $\mathrm{n}=5$ ). Five of the ten castrated mice were treated daily with enzalutamide (10 mg/kg MDV3100 in 10\%DMSO/PBS) by oral gavage for 7 days, beginning five days after surgery. After the animals were sacrificed, half the tumor was formalin-fixed and paraffin embedded and half was analyzed for lipidomics.

\section{shELOVL5 animal studies}

Subcutaneous and intraprostatic xenograft studies were approved by the University of Adelaide Animal Ethics Committee (approval number M-2016-160). Eight-week-old male 
NOD/SCID mice (Animal Resources Centre, Western Australia) received intraprostatic injections $\left(1 \times 10^{6}\right.$ shCTRL or shELOVL5 LNCaP cells in $10 \mu \mathrm{L}$ of PBS) or subcutaneous flank injections $\left(2 \times 10^{6}\right.$ shCTRL or shELOVL5 LNCaP cells in $50 \mu \mathrm{L}$ of PBS mixed with 50 $\mu \mathrm{L}$ Matrigel ${ }^{\mathrm{TM}}-\mathrm{HC}$ ). Each study used 7 animals per group. Noninvasive, whole-body imaging to monitor luciferase-expressing cells was performed at the time of injection and once weekly using the IVIS Spectrum In Vivo Imaging System (PerkinElmer) as described previously (18). Caliper measurements were taken regularly throughout the study. After the animals were sacrificed, internal organs and prostate glands were excised for ex vivo imaging using the IVIS system.

\section{$\underline{\text { Histological staining of mouse lung metastatic lesions }}$}

Hematoxylin and Eosin staining was performed on 2uM sections that were stained with hematoxylin (Australian Biostain), blued with 1\% acid alcohol and counterstained with eosin (Australian Biostain). Immunohistochemistry was performed on 2uM sections that were blocked for endogenous peroxidase before being subjected to heat-induced epitope retrieval. Sections were blocked in 10\% normal goat serum (Sigma) and incubated with AR primary antibody (1:200, Abcam ab108341) overnight, biotinylated anti-rabbit secondary antibody (1:400, DAKO) for $1 \mathrm{~h}$, and streptavidin-horseradish peroxidase complex (1:500, DAKO) for $1 \mathrm{~h}$. Positive cells were visualized with $D A B$ and counterstained with hematoxylin (Australian Biostain). Images were captured on a NanoZoomer digital slide scanner (Hamamatsu).

\section{Statistical Analysis}

Statistical analysis was carried out using GraphPad Prism software v7.02 (2016, GraphPad Software) and data are representative of at least 2 independent experiments. Bar graphs represent the mean \pm SEM of at least 3 biological replicates unless otherwise indicated. Dot plots represent the mean \pm SEM of individually counted cells, spheroids or 
lipid droplets from at least 3 biological replicates unless otherwise indicated. Box plots represent the mean \pm interquartiles, and whiskers represent minimum and maximum values. For cell line and animal studies, significance was measured by two-tailed unpaired $t$-test or one-way ANOVA with Dunnett's multiple comparison test as indicated. For clinical data, significance was measured using Mann-Whitney U-test. Significance is expressed as ${ }^{\star} P<0.05,{ }^{\star *} P<0.01$ and ${ }^{* * *} P<0.001$. Heat maps, hierarchical clustering and Pearson $\begin{array}{llll}\text { correlations } & \text { were } & \text { generated } & \text { using }\end{array}$ (www.broadinstitute.org/cancer/software/GENE-E/). All heat maps shown are rownormalized for presentation purposes. Pearsons correlation with average linkage was used for clustering. 


\section{Results}

Androgens induce fatty acyl chain elongation of phospholipids in prostate cancer cell lines

To investigate the influence of androgens on the prostate cancer lipidome, lipids extracted from androgen treated AR-positive LNCaP prostate cancer cells were subjected to quantitative lipidomic profiling by tandem mass spectrometry (Figure 1A). In cells treated with low $(1 \mathrm{nM})$ and high (10nM) physiological doses of the synthetic androgen mibolerone, total phospholipid abundance increased dose dependently compared to vehicle treated cells (Figure 1B). Analysis of the four major phospholipid classes, phosphatyidyl-choline $(\mathrm{PC})$, -ethanolamine $(\mathrm{PE})$, -inositol $(\mathrm{PI})$ and -serine (PS), revealed an increase in PC abundance with the low dose of $1 \mathrm{nM}$ mibolerone, and marked increases in abundance across all four classes with the high dose of $10 \mathrm{nM}$ mibolerone compared to vehicle (Figure 1C). Androgen treatment did not alter saturated fatty acid (SFA) levels but significantly increased the abundance of monounsaturated fatty acids (MUFAs - defined as phospholipids containing 1-2 unsaturations, i.e., one for each fatty acid tail) and polyunsaturated fatty acids (PUFAs - defined as phospholipids containing 3 or more unsaturations) at the high dose of 10nM mibolerone compared to vehicle (Figure 1D).

The most prominent effect of androgens on the lipidome was elongation of MUFAs and PUFAs across all four phospholipid classes analyzed. Elongation was evident as decreased abundance of short chain phospholipid species and a concomitant increase in long chain phospholipids with a given number of double bonds, and was observed in LNCaP cells treated with either $1 \mathrm{nM}$ mibolerone compared to vehicle (Supplementary Figure 1) or $1 \mathrm{nM}$ of the native androgen 5a-dihydrotestosterone (DHT) (Figure 1E, Supplementary Figure 1). The opposite effect on phospholipid chain length was observed LNCaP cells treated with the clinical AR antagonist enzalutamide (Figure 1E, Supplementary Figure 1). Androgen-induced elongation was also observed in AR-positive 
LAPC4 and 22Rv1 prostate cancer cells, but not in AR-negative PC-3 prostate cancer cells (Supplementary Figure 2A), which is consistent with the low level of ELOVL5 expression observed in PC-3 cells compared with AR-positive cell lines (Supplementary Figure 2B). The clinical relevance of this androgenic effect was addressed by undertaking phospholipidomic profiling in matched prostate cancer patient-derived explants (PDEs) treated with enzalutamide or vehicle ( $n=19$; Figure 1F). The major trend observed for the majority $(17 / 19 ; 89.4 \%)$ of enzalutamide-treated PDEs was inhibition of fatty acid chain elongation in MUFAs and PUFAs with up to 4 unsaturations (Figure 1G, Supplementary Figure 3). Taken together, we have demonstrated that androgens have a significant effect on phospholipid composition in prostate cancer cells and tumors, whereby androgens induce and enzalutamide inhibits fatty acid chain elongation (Figure 1H).

Androgen receptor directly regulates genes involved in fatty acid chain elongation

Consistent with the effects of androgens on fatty acyl chain length, we observed androgenic induction of three ELOVL fatty acid elongases (ELOVL) in LNCaP, LAPC4 and 22Rv1 prostate cancer cells, as well as induction of the known AR regulated gene FASN (Figure 2A). The most marked androgen-induced increases were observed for ELOVL2, ELOVL5 and ELOLV7, which was also evident in an independent, published LNCaP RNAseq dataset (19) (Figure 2B). Expression of these three ELOVLS was strongly associated with androgen-induced prostate cancer gene sets (Nelson, Figure 2C; Wang, Supplementary Figure 4A) (20,21). As expected, the AR antagonist enzalutamide significantly decreased ELOVL2, ELOVL5 and ELOLV7 expression compared to vehicle in LNCaP cell lines (Figure 2D) and LNCaP xenograft tumors (Figure 2E). Expression of ELOVL2 was not detected in the LNCaP xenografts. Importantly, ELOVL2, ELOVL5 and ELOVL7 were repressed by androgen deprivation in prostatic biopsies from patients with locally advanced or metastatic prostate cancer after short-term neoadjuvant ADT ( $n=7$; 
Figure 2F) (22), and in radical prostatectomy samples from neoadjuvant intense ADT patients ( $n=18$; Supplementary Figure 4B) (23). Previous studies suggested that androgens may mediate ELOVL expression indirectly through activation of the master lipogenic transcription factor, sterol regulatory element-binding protein (SREBP1) (24), or directly via AR transcriptional activity (25). To explore regulation through SREBP1, we knocked down SREBP1 in LNCP cells in the presence and absence of androgens (Supplementary Figure 4C; 84\% decrease in gene expression with siSREBP1 compared to siCTRL). As expected, siSREBP1 inhibited androgen induction of FASN expression, but was unable to suppress androgen induction of ELOVL2, ELOVL5 or ELOVL7 (Supplementary Figure 4C), which indicates that SREBP1 is not the primary mediator of androgen induced ELOVL expression. To explore direct AR regulation, we examined AR ChIP-seq data from clinical samples (13). Multiple AR binding sites proximal to the ELOVL2, ELOVL5 and ELOVL7 genes were evident in human prostatic tissues, many of which were enriched in malignant compared to normal tissues (Figure 2G; Supplementary Figure 5). Supporting their relevance, these AR binding sites were coincident with histone H3 lysine 27 acetylation (H3K27ac), a mark of active promoters and enhancers. We provide evidence of these AR binding sites in many published ChIP-seq datasets using ChIP-Atlas (Supplementary Figure 6A, 6B, 6C), and validated AR binding at a subset of these sites by ChIP-qPCR in LNCaP cells (Figure 2H). Collectively, these data indicate that $A R$ binds to multiple sites proximal to the transcriptional start sites of the ELOVL2, ELOVL5 and ELOVL7 genes, supporting a direct mode of AR-mediated transcriptional regulation.

ELOVL5 is the major elongase in the prostate and is overexpressed in prostate cancer

To assess the relative contributions of ELOVL2, ELOVL5 and ELOVL7 to the androgeninduced elongation phenotype, siRNA-mediated knockdown of all three ELOVLs in 
combination or alone was performed in LNCaP cells. Two independent siRNAs were tested per ELOVL gene in LNCaP cells, each achieving at least 80\% knockdown within 48 hours (Supplementary Figure 7A). We observed no evidence of ELOVL2, ELOVL5 or ELOVL7 knockdown alone causing compensatory increases in expression of the other ELOVLs (Figure $3 \boldsymbol{A}$ ). When compared with siCTRL, complete reversal of the androgenmediated increase in longer fatty acid chains was observed in all four phospholipid classes with simultaneous knockdown of ELOVL2/5/7 in LNCaP cells (Figure 3B, Supplementary Figure 7B). Notably, almost identical changes to the combined ELOVL2/5/7 knockdown were achieved by ELOVL5 knockdown alone (Figure 3B, Supplementary Figure 7B). ELOVL2 and ELOVL7 knockdown showed no discernible effect, implicating ELOVL5 as the primary contributor to androgen regulation of phospholipid fatty acyl chain elongation in LNCaP cells. Supporting ELOVL5 as the major elongase in prostate cancer, ELOVL5 was the most highly expressed of all seven ELOVL genes in two independent transcriptomic datasets, derived from 419 localized primary prostate tumors generated by the Cancer Genome Atlas (TCGA) Research Network (http://cancergenome.nih.gov/) (Figure 3C) and 118 metastatic castration resistant prostate tumors from the Stand up to Cancer (SU2C) dataset (Figure 3D). Given the high level of ELOVL5 expression in prostate cancer, we investigated whether this is aberrant expression compared to normal prostate tissue. Data from three clinical cohorts were evaluated, including transcriptomic datasets from Memorial Sloan-Kettering Cancer Center (MSKCC) (Figure 3E), TCGA (Figure 3F) and a proteomic dataset from Tampere University Hospital (26) (Supplementary Figure 8A). In all three cohorts, ELOVL5 expression was significantly higher in prostate cancer compared to normal/benign prostatic hyperplasia (BPH). Further supporting these observations, ELOVL5 protein levels were analyzed by immunohistochemistry in five tissue microarrays (TMAs) comprised of 249 primary prostate cancer samples. DAPI-normalized ELOVL5 intensity was significantly increased in tumor compared to adjacent benign prostate 
epithelial cells in the entire cohort (Figure $\mathbf{3 G - H}$ ), or when each TMA was independently evaluated (Supplementary Figure 8B).

\section{ELOVL5 targeting inhibits prostate cancer cell proliferation}

The significant role of ELOVL5 in modifying the cellular lipidome suggests that it may contribute to cellular proliferation and energetic processes. Indeed, transient knockdown of ELOVL5 by two independent siRNAs in LNCaP (Supplementary Figure 7A) and 22Rv1 cells (Supplementary Figure $8 \mathrm{C}$ ) significantly attenuated viability of both prostate cancer cell lines (Figure 4A-B), but did not induce death (Figure 4A-B) or apoptosis (Supplementary Figure 8D). In contrast, constitutive overexpression of ELOVL5 (hELOVL5+) in LNCaP cells significantly increased viable cell number compared to control cells (hCTRL; Figure 4C). LNCaP colony formation was affected in a similar manner, where stable knockdown of ELOVL5 with short hairpin RNA (shELOVL5-) significantly reduced colony formation (Figure 4D) while stable overexpression of ELOVL5 increased colony formation (Figure 4E). Given we observed no impact of ELOVL5 depletion on cell death, we evaluated proliferation as the driver of ELOVL5-mediated increase in cell viability. Transient knockdown of ELOVL5 with siRNA significantly attenuated LNCaP and 22Rv1 cell proliferation compared to control siRNA, as determined by a Cyquant assay (Figure 4F), which was associated with cell cycle arrest at the G1/S phase (Figure 4G). Encouraged by the anti-proliferative effects of ELOVL5 knockdown in prostate cancer cell lines, we again used our PDE model to determine efficacy of ELOVL5 targeting in clinical prostate tumors. Significant ELOVL5 depletion (44.7\%) was achieved in PDE tissues using siRNA loaded nanoparticles (Figure $\mathbf{4 H}$, Supplementary Figure $8 \mathrm{E}$ ), and this resulted in a marked decrease in expression of the proliferative marker ki67 from $52.9 \%$ in siCTRL PDE tissues to $21.06 \%$ in siELOVL5 PDE tissues (Figure $\mathbf{4 H}$, Supplementary Figure $8 \mathrm{E}$ ). Finally, reduced spheroid growth was observed in siELOVL5-depleted LNCaP cells 
compared to siCTRL (Figure 4I), and constitutively expressing shELOVL5 LNCaP cells showed significantly reduced capacity for tumor growth compared to ShCTRL cells when grown subcutaneously in NOD/SCID mice ( $n=7$ mice/treatment) (Figure 4ل).

ELOVL5 elongation of the monounsaturated fatty acid cis-Vaccenic acid is critical for prostate cancer cell viability

Published phenotypic studies of ELOVL5 knockout mice, demonstrate that hepatic steatosis induced by ELOVL5 knockout can be rescued by supplementation with the PUFAs arachidonic acid (AA; C20:4,n-6) and docosahexaenoic acid (DHA; C22:6,n-3) (27), as these PUFAs are reliant on ELOVL5 for synthesis (Figure 5A). We found that although AA and DHA levels were reduced in ELOVL5-depleted LNCaP cells (Supplementary Figure 9A), supplementation with AA or DHA did not alter ELOVL5 knockdown-mediated changes in cell viability (Figure 5B). Also distinct from the mouse model, ELOVL5 knockdown caused no induction of key lipid metabolism enzymes (SREBP1, FASN, ACACA and ATGL; Supplementary Figure 9B), nor any alteration to cellular lipid content, as determined by the number of lipid droplets observed per cell by electron microscopy (Supplementary Figure 9C). These findings indicated that the role/s of ELOVL5 in prostate cells differ substantially from those in the liver. In considering other known products of ELOVL5, we tested an omega-7 monounsaturated fatty acid (MUFA), cis-vaccenic acid (cVA; C18:1,n-7), which has previously been implicated in ELOVL5 action (Figure 5A) (28). Remarkably, supplementation with cVA completely reversed the inhibitory effect of ELOVL5 knockdown on cell viability (Figure 5 C), indicating MUFA elongation as a key ELOVL5 function in prostate cancer.

ELOVL5 activity regulates mitochondrial respiratory function and oxidative stress 
Electron microscopy examination of ELOVL5-depleted LNCaP cells showed a marked increase in the size and length of the cellular mitochondria compared to control cells, as well as darker and denser cristae (Figure 6A), indicative of cellular stress. Further investigation of the mitochondria was undertaken using the Seahorse mitochondrial stress test assay. Decreased basal and maximal respiration rates were observed in ELOVL5depleted LNCaP cells (Figure 6 B), whereas ELOVL5 overexpression increased maximal mitochondrial respiration (Figure 6C). A decrease in mitochondrial respiration, as observed upon ELOVL5 knockdown, is a strong indicator of mitochondrial dysfunction and can result in aberrant generation of reactive oxygen species (ROS) (29). The fluorogenic dye CellROX ORANGE was used to investigate whether ELOVL5 targeting influences ROS production. Markedly increased ROS levels were observed upon ELOVL5 knockdown in LNCaP cells, which was completely reversed by the potent antioxidant/ROS scavenger Trolox (Figure 6D). Trolox also rescued the inhibitory effects of ELOVL5 depletion on LNCaP cell viability (Figure 6E), revealing ROS production as a key antiproliferative mechanism for ELOVL5 targeting. Consistent with these results, ELOVL5mediated changes in ROS production were also rescued by supplementation with cVA (Figure 6E). Confirming that ROS induction was not part of a generalized stress response, stable overexpression of ELOVL5 markedly reduced ROS levels in LNCaP cells (Figure $6 F)$.

\section{ELOVL5 promotes prostate tumor growth and metastasis}

Having established a critical role for ELOVL5 in prostate cancer cell proliferation, we next investigated its influence on metastatic tumor features in vitro and in vivo. ELOVL5 knockdown significantly reduced transwell migration through Matrigel in both LNCaP (Figure 7A) and 22Rv1 cells (Figure 7B), whereas ELOVL5 overexpression promoted LNCaP migration (Figure 7C). Importantly, supplementation of ELOVL5-depleted LNCaP 
cells with cVA reversed the migratory phenotype (Figure 7D), reinforcing a critical role for this MUFA in prostate cancer. To determine whether ELOVL5 influences spontaneous metastasis, we employed an orthotopic assay in which LNCaP cells were injected directly into the prostate (i.e., intraprostatic xenografts). Weekly monitoring of the mice revealed that tumor incidence was $100 \%$ for both shELOVL5 and shCTRL cells, but the shELOVL5 tumors grew significantly more slowly and measured almost $40 \%$ smaller than shCTRL tumors after 50 days (Figure 7E). Ex vivo bioluminescent imaging of the major organs following intraprostatic tumor growth revealed that shCTRL cells spread to major visceral organs, most notably the lungs, in 4 of 7 mice (Figure 7F). Histological evidence of LNCaP tumor metastases to the lung was demonstrated by staining for AR, GFP and H\&E (Figure 7F). Significantly, no overt metastasis by shELOVL5 cells were detected in any mice (Figure 7F). 


\section{Discussion}

The advent of high throughput 'omics' approaches has revolutionized the molecular characterization of cancer, but in contrast to the genome, transcriptome and proteome, the lipidome remains largely underexplored. We utilized tandem mass spectrometric analysis to profile the androgen-regulated lipidome of prostate cancer cell lines and patient-derived explants and uncovered ubiquitous increases in fatty acyl chain elongation mediated by the fatty acid elongase, ELOVL5. Molecular targeting of ELOVL5 reversed fatty acyl chain elongation in prostate cancer cells, which through induction of mitochondrial oxidative stress, significantly reduced prostate tumorigenesis and metastatic potential in vitro and in vivo. Moreover, we demonstrate that the monounsaturated fatty acid product of ELOVL5 activity, cVA, is a critical modulator of ELOVL5 induced oxidative stress and survival in prostate cancer. Our findings provide new insights into the downstream metabolic actions of $A R$ in prostate cancer cells, beyond the previous almost exclusive focus on de novo lipogenesis, and identify fatty acid elongation as a critical target of androgen action for therapeutic intervention.

Elongation is a critical step in the production of fatty acids with a chain length of more than 16 carbons, and is a stepwise process whereby carbon units are added to the carboxyl terminal of fatty acid chains (30). The reaction is catalyzed by enzymes called elongases, encoded by a family of seven ELOVL genes (ELOVL1-7) that display distinct tissue distribution and substrate preference (31). ELOVL5 is most highly expressed in the adrenal glands, testis and prostate (32). Increasing evidence suggests that ELOVL expression is frequently altered in cancer. ELOVL6 is the most commonly studied, with reported overexpression in hepatocellular cancer (33), lung cancer (34) and breast cancer (35). In prostate cancer, ELOVL7 has been reported as overexpressed in hormone-naïve prostate cancer $(36,37)$, with influences on cell viability, xenograft tumor growth and 
potentially de novo steroidogenesis $(36,38)$, however we found its abundance in clinical prostate tumors to be very low. When directly comparing all seven ELOVL family members at both the RNA and protein level, ELOVL5 was the most highly expressed in both primary and castration resistant clinical prostate tumors, and was overexpressed in malignant compared to non-malignant prostate. In published overexpression studies in primary rat hepatocytes, adenoviral overexpression of ELOVL5 induced elongation of C20 PUFAs and gave rise to altered hepatic fatty acid content (39). The reverse was observed in ELOVL5 knock out mice where inhibition of C20 and C22 PUFA elongation and concomitant increase in C18 PUFAs was observed, and resulted in development of hepatic steatosis (27). Unfortunately, the effect of ELOVL5 ablation on the mouse prostate was not reported, but we likewise observed inhibition of fatty acid elongation in prostate cancer cells with ELOVL5 knockdown. Phenotypically, targeting ELOVL5 had profound effects on prostate cancer cell proliferation, colony formation, spheroid growth and invasion through Matrigel, in pre-clinical models that range from typical cell lines to complex 3D-cultures and patientderived explants. The latter provides the most physiologically and clinically relevant preclinical model currently available, as the critical tumor microenvironment remains intact (10). The therapeutic efficacy of ELOVL5 depletion was also significant in vivo where primary tumor growth was suppressed and spontaneous metastasis to distant sites was restricted by the knock down.

Demonstrated in this study is a previously unknown relationship between ELOVL5-driven lipid elongation in prostate cancer, mitochondrial homeostasis and intracellular ROS levels. ELOVL5's dual effects on mitochondrial respiratory activity and intracellular ROS levels are consistent, given mitochondria are a major source of intracellular ROS (40). Importantly, our data indicates that ROS induction is not a non-specific cellular stress response to ELOVL5 targeting, as ectopic ELOVL5 overexpression markedly suppresses ROS production. It is well established that ROS production is elevated in cancer cells, as a 
consequence of increased metabolic demand, mutation rate and hypoxia, as a means of promoting cancer cell proliferation and tumor progression (41). While these effects are beneficial for cancer cell growth and survival, the increase in baseline ROS also represents a vulnerability to further oxidative stress, as high levels of ROS are cytotoxic (42). Unlike their benign counterparts with low baseline ROS levels, small increases in ROS production can push cancer cells over the "cytotoxic ROS threshold" (41) to suppress cell survival and/or induce cell death. The role of ELOVL5 in prostate cancer appears to be, at least in part, as a regulator of cellular redox homeostasis, to ensure that prostate cancer cells do not exceed this "cytotoxic ROS threshold", whilst maintaining appropriate ROS production to drive tumorigenesis. While an increasing number of cancer treatments target ROS as a therapeutic strategy, including both chemotherapies and molecular targeted agents (43), specific targeting of lipid metabolism through ELOVL5 now provides a new avenue for ROS induction as a treatment for prostate cancer.

One of the most significant findings of this study was rescue of ELOVL5-mediated proliferation, metastasis and ROS induction by supplementation with the monounsaturated product of ELOVL5, cis-vaccenic acid, but not by supplementation with long-chain polyunsaturated fatty acids. This was an unexpected, since ELOVL5 was previously considered to be mainly involved in production of PUFAs rather than MUFAs, and the main hepatic phenotype of the ELOVL5 knockout mouse was fully rescued by PUFA supplementation. In light of recent reports of the critical importance of MUFAs in maintaining redox homeostasis in cancer cells (44), our data suggest that cis-vaccenic acid production underpins this aspect of ELOVL5 activity, and may serve to reduce oxidation of ELOVL5-derived PUFAs. While it is the least studied of ELOVL5's products, cis-vaccenic acid has been reported to be linked to coronary heart disease risk when incorporated into red blood cell membranes (45), has displayed anti-lipogenic effects in bovine adipocytes (46) and its ratio with its precursor has been suggested as an indicator 
of biotin deficiency (47). A study undertaken in HepG2 (kidney) cells suggested that cisvaccenic was the mediator of ELOVL5 regulation of the mTORC2-Akt-FOXO1 pathway (28). Importantly, its role in metabolism is largely unknown (46) and there is little to no published data on its role in cancer, highlighting this as an important focus for future investigations.

Important for the potential of ELOVL5 as a therapeutic target, the murine ELOVL5 knock out was not lethal. This is in contrast to the master fatty acid synthesis enzyme FASN, which is lethal when knocked out in mice (48), and suggests that targeted inhibition of ELOVL5 could have a higher therapeutic window than inhibition of FASN - currently the most widely studied metabolic target in cancer. Moreover, loss of ELOVL5 did not elicit changes to the expression of other ELOVL family members, key lipid synthetic enzymes or to cellular fatty acid content. These findings further highlight the potential of ELOVL5 as a therapeutic target, as compensatory adaptive responses that can mitigate efficacy have frequently been observed with investigative metabolic drugs and limit their clinical application. For example, FASN inhibitors target de novo lipid synthesis but can result in compensatory increases in uptake of exogenous dietary lipids or expression of genes in the fatty acid synthesis pathway including ACACA, ACLY as well as FASN itself, to satisfy the cell's demand for fatty acids $(49,50)$.

Transcriptomic studies have suggested for many years that lipid synthesis is enriched by AR activity in prostate cancer but the explicit details of how this contributes to tumorigenicity have remained unclear. Herein we provide a specific new functional role for the AR in maintaining lipid homeostasis via ELOVL5-mediated elongation of fatty acids, and demonstrate the significant impact of elongation on prostate cancer cell malignancy and metastasis. The anti-metastatic effects of ELOVL5 knockdown are particularly notable given that metastatic prostate cancer remains a major clinical challenge and is associated 
with a high rate of death despite multiple new drug approvals in recent years. Based on the findings in this study, targeting specific lipidomic features of prostate tumor cells such as elongation is a promising therapeutic strategy that warrants further investigation. 


\section{Acknowledgements}

The authors thank the study participants, urologists, nurses, histopathologists, and the South Australian Coordinator of the APCC, Ms. Jessica Savage, who assisted in the recruitment and collection of patient material and information. Ms Swati Irani for assistance with explant tissue culture and analysis. Dr Ying Huang for immunostaining the tissue microarrays. The University of Adelaide Microscopy facility for technical assistance with electron microscopy. The authors acknowledge financial support from the Movember Foundation and Prostate Cancer Foundation of Australia (MRTA3 to LM Butler, JV Swinnen, WD Tilley, AM Scott, LA Selth, MM Centenera; MRTA1 to ED Williams), Prostate Cancer Foundation of Australia (NDDA2711 to LM Butler, MM Centenera, JV Swinnen; NCG1816 to LM Butler, A Cifuentes-Rius, NH Voelcker), the Research Foundation Flanders (FWO G.0841.15 to JV Swinnen), the Stichting tegen Kanker (to JV Swinnen), KU Leuven (C16/15/073 and C32/17/052 to JV Swinnen), Interreg V-A (EMR23 to JV Swinnen and E Waelkens), the National Health and Medical Research Council (1121057 to WD Tilley, LA Selth), the National Institute of Health (RO1CA131945 to M Loda), the National Cancer Institute (P50CA211024 to M Loda), the Prostate Cancer Foundation (to M Loda), the United States Department of Defense (PC150263 to G Zadra, PC160357 to M Loda, PC180582 to M Loda, LM Butler, JV Swinnen), and the Dana-Farber Cancer Institute (Claudia Adams Barr Award in Innovative Basic Cancer Research to G Zadra). MM Centenera was supported by a Prostate Cancer Foundation of Australia Young Investigator Award (YIG0412). ZD Nassar was supported by National Health and Medical Research Center Early Career Fellowship (1138648) and Prostate Cancer Foundation of Australia John Mills Young Investigator Award (Yl1417). T Tieu and NH Voelcker acknowledge support from the Office of the Chief Executive and CSIRO Manufacturing. T Tieu acknowledges support of an Australian Government RTP scholarship. A CifuentesRius was supported by a National Health \& Medical Research Council Early Career 
Fellowship (1112432). LM Butler was supported by an ARC Future Fellowship (130101004). LM Butler and LA Selth are supported by Principal Cancer Research Fellowships awarded by Cancer Council's Beat Cancer project on behalf of its donors, the state Government through the Department of Health, and the Australian Government through the Medical Research Future Fund (PRF1117 and PRF2919).

\section{Author Contributions}

Conceptualization; MMC, JVS, LMB. Investigation; MMC, JM, ZDN, IZ, JD, JS, IJGD, GZ, PMC, CB, EE, NKR, JLG, CYM, TT, AH, DM, RC, KB, VP, EW, RD, EDW, AE. Writing Original Draft; MMC. Writing - Review \& Editing; MMC, ACR, NHV, IGM, WDT, AMS, ML, LAS, JVS, LMB. Supervision; JVS and LMB 


\section{References}

1. Siegel RL, Miller KD, Jemal A. Cancer statistics, 2016. CA Cancer J Clin 2016;66:7-30

2. Attard G, Parker C, Eeles RA, Schroder F, Tomlins SA, Tannock I, et al. Prostate cancer. Lancet 2016;387:70-82

3. Swinnen JV, Esquenet M, Goossens K, Heyns W, Verhoeven G. Androgens stimulate fatty acid synthase in the human prostate cancer cell line LNCaP. Cancer Res 1997;57:1086-90

4. Nguyen PL, Ma J, Chavarro JE, Freedman ML, Lis R, Fedele G, et al. Fatty acid synthase polymorphisms, tumor expression, body mass index, prostate cancer risk, and survival. J Clin Oncol 2010;28:3958-64

5. Swinnen JV, Roskams T, Joniau S, Van Poppel H, Oyen R, Baert L, et al. Overexpression of fatty acid synthase is an early and common event in the development of prostate cancer. Int J Cancer 2002;98:19-22

6. Rysman E, Brusselmans K, Scheys K, Timmermans L, Derua R, Munck S, et al. De novo lipogenesis protects cancer cells from free radicals and chemotherapeutics by promoting membrane lipid saturation. Cancer Res 2010;70:8117-26

7. Zadra G, Photopoulos C, Loda M. The fat side of prostate cancer. Biochim Biophys Acta 2013;1831:1518-32

8. Swinnen JV, Heemers H, van de Sande T, de Schrijver E, Brusselmans K, Heyns W, et al. Androgens, lipogenesis and prostate cancer. J Steroid Biochem Mol Biol 2004;92:273-9

9. Sharma NL, Massie CE, Ramos-Montoya A, Zecchini V, Scott HE, Lamb AD, et al. The Androgen Receptor Induces a Distinct Transcriptional Program in Castration-Resistant Prostate Cancer in Man. Cancer Cell 2013;23:35-47

10. Centenera MM, Hickey TE, Jindal S, Ryan NK, Ravindranathan $\mathrm{P}$, Mohammed $\mathrm{H}$, et al. A patientderived explant (PDE) model of hormone-dependent cancer. Mol Oncol 2018;12:1608-22

11. Selth LA, Das R, Townley SL, Coutinho I, Hanson AR, Centenera MM, et al. A ZEB1-miR-375-YAP1 pathway regulates epithelial plasticity in prostate cancer. Oncogene 2017;36:24-34

12. Taylor BS, Schultz N, Hieronymus H, Gopalan A, Xiao Y, Carver BS, et al. Integrative genomic profiling of human prostate cancer. Cancer Cell 2010;18:11-22

13. Pomerantz MM, Li F, Takeda DY, Lenci R, Chonkar A, Chabot M, et al. The androgen receptor cistrome is extensively reprogrammed in human prostate tumorigenesis. Nat Genet 2015;47:134651

14. Freese $\mathrm{NH}$, Norris $\mathrm{DC}$, Loraine AE. Integrated genome browser: visual analytics platform for genomics. Bioinformatics 2016;32:2089-95

15. Paltoglou S, Das R, Townley SL, Hickey TE, Tarulli GA, Coutinho I, et al. Novel Androgen Receptor Coregulator GRHL2 Exerts Both Oncogenic and Antimetastatic Functions in Prostate Cancer. Cancer Res 2017;77:3417-30

16. Tieu T, Dhawan S, Haridas V, Butler LM, Thissen H, Cifuentes-Rius A, et al. Maximizing RNA Loading for Gene Silencing Using Porous Silicon Nanoparticles. ACS applied materials \& interfaces 2019;11:22993-3005

17. Centenera MM, Gillis JL, Hanson AR, Jindal S, Taylor RA, Risbridger GP, et al. Evidence for efficacy of new Hsp90 inhibitors revealed by ex vivo culture of human prostate tumors. Clin Cancer Res 2012;18:3562-70

18. Panagopoulos V, Leach DA, Zinonos I, Ponomarev V, Licari G, Liapis V, et al. Inflammatory peroxidases promote breast cancer progression in mice via regulation of the tumour microenvironment. Int J Oncol 2017;50:1191-200

19. Spans L, Helsen C, Clinckemalie L, Van den Broeck T, Prekovic S, Joniau S, et al. Comparative genomic and transcriptomic analyses of $\mathrm{LNCaP}$ and $\mathrm{C} 4-2 \mathrm{~B}$ prostate cancer cell lines. PloS one 2014;9:e90002

20. Nelson PS, Clegg N, Arnold H, Ferguson C, Bonham M, White J, et al. The program of androgenresponsive genes in neoplastic prostate epithelium. Proc Natl Acad Sci U S A 2002;99:11890-5

21. Wang G, Jones SJ, Marra MA, Sadar MD. Identification of genes targeted by the androgen and PKA signaling pathways in prostate cancer cells. Oncogene 2006;25:7311-23 
22. Rajan P, Sudbery IM, Villasevil ME, Mui E, Fleming J, Davis M, et al. Next-generation sequencing of advanced prostate cancer treated with androgen-deprivation therapy. Eur Urol 2014;66:32-9

23. Sowalsky AG, Ye H, Bhasin M, Van Allen EM, Loda M, Lis RT, et al. Neoadjuvant-Intensive Androgen Deprivation Therapy Selects for Prostate Tumor Foci with Diverse Subclonal Oncogenic Alterations. Cancer Res 2018;78:4716-30

24. Heemers HV, Verhoeven G, Swinnen JV. Androgen activation of the sterol regulatory elementbinding protein pathway: Current insights. Mol Endocrinol 2006;20:2265-77

25. Chan SC, Selth LA, Li Y, Nyquist MD, Miao L, Bradner JE, et al. Targeting chromatin binding regulation of constitutively active $A R$ variants to overcome prostate cancer resistance to endocrinebased therapies. Nucleic Acids Res 2015;43:5880-97

26. Latonen L, Afyounian E, Jylha A, Nattinen J, Aapola U, Annala M, et al. Integrative proteomics in prostate cancer uncovers robustness against genomic and transcriptomic aberrations during disease progression. Nat Commun 2018;9:1176

27. Moon YA, Hammer RE, Horton JD. Deletion of ELOVL5 leads to fatty liver through activation of SREBP-1c in mice. J Lipid Res 2009;50:412-23

28. Tripathy S, Jump DB. Elovl5 regulates the mTORC2-Akt-FOXO1 pathway by controlling hepatic cisvaccenic acid synthesis in diet-induced obese mice. J Lipid Res 2013;54:71-84

29. Murphy MP. How mitochondria produce reactive oxygen species. Biochem J 2009;417:1-13

30. Kihara A. Very long-chain fatty acids: elongation, physiology and related disorders. J Biochem 2012;152:387-95

31. Jakobsson A, Westerberg R, Jacobsson A. Fatty acid elongases in mammals: their regulation and roles in metabolism. Prog Lipid Res 2006;45:237-49

32. Leonard AE, Bobik EG, Dorado J, Kroeger PE, Chuang LT, Thurmond JM, et al. Cloning of a human cDNA encoding a novel enzyme involved in the elongation of long-chain polyunsaturated fatty acids. Biochem J 2000;350 Pt 3:765-70

33. Matsuzaka $\mathrm{T}$, Atsumi A, Matsumori R, Nie T, Shinozaki $\mathrm{H}$, Suzuki-Kemuriyama $\mathrm{N}$, et al. Elovl6 promotes nonalcoholic steatohepatitis. Hepatology 2012;56:2199-208

34. Marien E, Meister M, Muley T, Gomez Del Pulgar T, Derua R, Spraggins JM, et al. Phospholipid profiling identifies acyl chain elongation as a ubiquitous trait and potential target for the treatment of lung squamous cell carcinoma. Oncotarget 2016;7:12582-97

35. Feng YH, Chen WY, Kuo YH, Tung CL, Tsao CJ, Shiau AL, et al. Elovl6 is a poor prognostic predictor in breast cancer. Oncol Lett 2016;12:207-12

36. Tamura K, Makino A, Hullin-Matsuda F, Kobayashi T, Furihata M, Chung S, et al. Novel lipogenic enzyme ELOVL7 is involved in prostate cancer growth through saturated long-chain fatty acid metabolism. Cancer Res 2009;69:8133-40

37. Tolkach Y, Merseburger A, Herrmann T, Kuczyk M, Serth J, Imkamp F. Signatures of Adverse Pathological Features, Androgen Insensitivity and Metastatic Potential in Prostate Cancer. Anticancer Res 2015;35:5443-51

38. Han W, Gao S, Barrett D, Ahmed M, Han D, Macoska JA, et al. Reactivation of androgen receptorregulated lipid biosynthesis drives the progression of castration-resistant prostate cancer. Oncogene 2018;37:710-21

39. Wang Y, Torres-Gonzalez M, Tripathy S, Botolin D, Christian B, Jump DB. Elevated hepatic fatty acid elongase-5 activity affects multiple pathways controlling hepatic lipid and carbohydrate composition. J Lipid Res 2008;49:1538-52

40. Rodic S, Vincent MD. Reactive oxygen species (ROS) are a key determinant of cancer's metabolic phenotype. Int J Cancer 2018;142:440-8

41. Nogueira V, Hay N. Molecular pathways: reactive oxygen species homeostasis in cancer cells and implications for cancer therapy. Clin Cancer Res 2013;19:4309-14

42. Hayes JD, Dinkova-Kostova AT, Tew KD. Oxidative Stress in Cancer. Cancer Cell 2020;38:167-97

43. Perillo B, Di Donato M, Pezone A, Di Zazzo E, Giovannelli P, Galasso G, et al. ROS in cancer therapy: the bright side of the moon. Exp Mol Med 2020;52:192-203

44. Magtanong L, Ko PJ, To M, Cao JY, Forcina GC, Tarangelo A, et al. Exogenous Monounsaturated Fatty Acids Promote a Ferroptosis-Resistant Cell State. Cell Chem Biol 2019;26:420-32 e9 
45. Djousse L, Matthan NR, Lichtenstein AH, Gaziano JM. Red blood cell membrane concentration of cis-palmitoleic and cis-vaccenic acids and risk of coronary heart disease. Am J Cardiol 2012;110:53944

46. Burns TA, Kadegowda AK, Duckett SK, Pratt SL, Jenkins TC. Palmitoleic ( $16: 1$ cis-9) and cis-vaccenic (18:1 cis-11) acid alter lipogenesis in bovine adipocyte cultures. Lipids 2012;47:1143-53

47. Liu YY, Shigematsu Y, Bykov I, Nakai A, Kikawa Y, Fukui T, et al. Abnormal fatty acid composition of lymphocytes of biotin-deficient rats. J Nutr Sci Vitaminol 1994;40:283-8

48. Chirala SS, Chang H, Matzuk M, Abu-Elheiga L, Mao J, Mahon K, et al. Fatty acid synthesis is essential in embryonic development: fatty acid synthase null mutants and most of the heterozygotes die in utero. Proc Natl Acad Sci U S A 2003;100:6358-63

49. Sankaranarayanapillai M, Zhang N, Baggerly KA, Gelovani JG. Metabolic Shifts Induced by Fatty Acid Synthase Inhibitor Orlistat in Non-small Cell Lung Carcinoma Cells Provide Novel Pharmacodynamic Biomarkers for Positron Emission Tomography and Magnetic Resonance Spectroscopy. Mol Imaging Biol 2013;15:136-47

50. Alwarawrah Y, Hughes P, Loiselle D, Carlson DA, Darr DB, Jordan JL, et al. Fasnall, a Selective FASN Inhibitor, Shows Potent Anti-tumor Activity in the MMTV-Neu Model of HER2(+) Breast Cancer. Cell Chem Biol 2016;23:678-88 


\section{FIGURE LEGENDS}

Figure 1: Androgens influence fatty acid chain elongation of phospholipids in prostate cancer. (A) Schematic diagram of experimental design. Phospholipids were extracted from prostate cancer cells and treated \pm androgens for $72 \mathrm{~h}$ prior to mass spectrometry based lipidomics. (B-D) Phospholipidomic changes in total lipid abundance, abundance per lipid class and saturation in LNCaP cells following treatment with synthetic androgen mibolerone. Data is presented as mean \pm SEM of triplicate wells. ${ }^{* *} p<0.01 ;{ }^{* * *}$ $p<0.001 ;{ }^{* * *} p<0.0001 ;$ ANOVA treatment versus vehicle. (E) Phospholipidomic changes in LNCaP cells following treatment with androgen $5 \alpha$-dihydrotestosterone (DHT), or AR antagonist enzalutamide (Enz). Data is presented as log2 fold change in relative abundance of phosphatidylethanolamine (PE) lipid species in treated versus control cells $(n=3)$. Lipids are labelled along the $X$-axis as $X X: Y$ where $X X$ denotes the total number of fatty acid carbons and $Y$ the total number of unsaturations in the fatty acyl chains. Lipids are grouped according to degree of unsaturation (groups separated by dashed lines). Within each saturation group, lipids are ordered from left to right by increasing acyl chain length. (F) Schematic diagram of experimental design. Phospholipids were extracted from patient-derived prostate cancer explants treated \pm enzalutamide for $48 \mathrm{~h}$ prior to mass spectrometry based lipidomics. (G) Hierarchical clustered heat map represents log2 fold change in relative abundance of select phosphatidylcholine (PC) species in enzalutamide treated explants compared to vehicle $(n=19)$. Average log2 fold change in PC abundance depicted in histogram to highlight inhibition of elongation by enzalutamide in patientderived prostate cancer explants. (H) Androgens significantly influence phospholipid structure by inducing elongation of its fatty acyl tails, whereas enzalutamide shortens phospholipid fatty acid tails. 
Figure 2: Androgen receptor regulates genes involved in fatty acid elongation. (A) qRT-PCR analysis of key genes involved in lipid metabolism in LNCaP, 22Rv1 and LAPC4 prostate cancer cells stimulated with androgen ( $1 \mathrm{nM}$ mibolerone). Data is presented as mean \pm SEM of triplicate wells. ${ }^{*} p<0.05$; ANOVA treatments versus vehicle. (B) Bar graph representing RNA-seq analysis of ELOVL genes in LNCaP cells treated with androgen (1nM R1881) or vehicle control. Data is presented as mean \pm SEM of triplicate wells. ${ }^{*} p<0.05$; ANOVA treatments versus vehicle. (C) ELOVL2, ELOVL5 and ELOVL7 expression is positively correlated with genes induced by $\mathrm{DHT}$ in prostate cancer cells, as assessed by GSEA. DHT-induced genes in the ordered data set are shown as black lines in the middle, and the running enrichment score is plotted at the top. (D) qRT-PCR analysis of all ELOVL genes in LNCaP cells stimulated with the AR antagonist enzalutamide $(1 \mu \mathrm{M}$ and $10 \mu \mathrm{M})$ compared to vehicle control treated cells. Data is presented as mean \pm SEM of triplicate wells. ${ }^{*} p<0.05$; ANOVA treatment versus vehicle. (E) qRT-PCR analysis of ELOVL2 (ND; not detected), ELOVL5, ELOVL7 and known androgen-regulated genes FKBP5 and KLK2 in subcutaneous LNCaP xenograft tumors obtained from mice treated with vehicle control $(n=5)$ or enzalutamide $(E N Z ; n=5)$. ${ }^{*} \mathrm{p}<0.05$; ANOVA treatments versus vehicle. (F) RNA-seq analysis of ELOVL2, ELOVL5 and ELOVL7 expression in prostate cancer patient material pre- and post- treatment with androgen deprivation therapy. (G) AR binding sites proximal to the ELOVL2, ELOVL5 and ELOVL7 genes in normal prostate tissues (mean ChIP-seq signal of 7 samples) and prostate tumors (mean ChIP-seq signal of 13 samples). (H) Validation of 3 putative AR binding sites (highlighted below the ChIP-seq tracks in Figure 3B) by ChIP-qPCR. Negative Control (NC). Data is presented as mean \pm SEM of triplicate wells.

Figure 3: ELOVL5 is the predominant elongase in prostate cells and is overexpressed in prostate cancer. (A) qRT-PCR analysis to determine specificity of siRNA directed to ELOVL2, ELOVL5 or ELOVL7 in LNCaP cells. Data is presented as fold 
change over siCTRL and error bars represent mean \pm SEM of triplicate wells. ${ }^{* \star * *} p<0.05$ ANOVA; siELOVL versus siCTRL. (B) Phospholipidomic changes in LNCaP cells subjected to knockdown of ELOVL2, ELOVL5 and ELOVL7 alone or in combination, in the presence of androgen ( $1 \mathrm{nM}$ mibolerone). Heat maps represents the log2 fold change in relative abundance of all measured PC species in androgen or siELOVL treated cells $(n=3)$. Red box depicts one unsaturation group as a bar graph to highlight effects of siELOVL on elongation. Data is presented as the mean \pm SEM of triplicate wells. ${ }^{* * *} p<0.0001$ ANOVA; short acyl chain lengths versus long acyl chain length, ns=not significant for any comparison. (C-D) RNA-seq analysis of ELOVL expression in primary prostate tumors (TCGA) and metastatic castration resistant prostate tumors (SU2C). (E-F) ELOVL5 transcript levels in normal prostate tissue compared to prostate cancer in two independent datasets (MSKCC and TCGA). Data is presented as normalized ELOVL5 expression. Boxplots show interquartiles with mean values, whiskers represent minimum and maximum values. ${ }^{*} \mathrm{p}<0.05$; Mann-Whitney test. (G) ELOVL5 protein expression in cancer compared to benign prostate clinical tissues in 5 primary prostate cancer tissue microarrays $(n=249)$. Violin plots are presented using normalized intensity units. ${ }^{*} p<0.001$; Mann-Whitney U-test. (H) Representative ELOVL5 immunostaining. Left panel shows multiplex fluorescence staining of ELOVL5 (magenta) in malignant glands as indicated by the absence of basal cells (red stain). Luminal cells are stained green. Chromogenic staining of ELOVL5 (brown stain) is shown in the right panels. Black arrow indicates benign prostate gland.

Figure 4: ELOVL5 knockdown inhibits prostate cancer cell growth, associated with impaired mitochondrial respiration and ROS production. Prostate cancer cell lines subjected to siRNA-mediated ELOVL5 knockdown (siELOVL5) or scrambled siRNA control (siCTRL) were analysed using the Trypan blue dye exclusion assay for (A) LNCaP cell viability and death, and (B) 22Rv1 cell viability and death. (C) LNCaP cells with stable 
overexpression of ELOVL5 (hELOVL5+) were analysed for cell viability compared to control cells (hCTRL). (D-E) LNCaP colony formation was evaluated in cells with stable shRNA ELOVL5 knockdown (shELOVL5) or stable ELOVL5 overexpression (hELOVL5+). (F) LNCaP and 22Rv1 cell proliferation was evaluated by Cyquant assay following siRNAmediated ELOVL5 knockdown. (G) LNCaP and 22Rv1 cell cycle distribution was evaluated by flow cytometric analysis $72 \mathrm{~h}$ after siRNA-mediated ELOVL5 knockdown. (H) Immunostaining for ELOVL5 and proliferative marker Ki67 in untreated, NPsiCTRL treated or NPsiELOVL5 treated patient-derived explants (PDEs). A decrease in proliferative marker Ki67 expression can be seen when PDE tissues are treated with NPsiELOVL5 $(n=6)$. (I) LNCaP spheroid volume was evaluated in LNCaP cells subjected to siRNAmediated ELOVL5 knockdown, compared to siCTRL. (J) Inhibition of subcutaneous tumor growth in stable ELOVL5 knockdown LNCaP cells (shELOVL5-) ( $n=7)$ compared to shCTRL LNCaP cells $(n=7)$ and representative bioluminescent tumor images. All in vivo data is representative of at least 3 independent experiments. Data is presented as mean \pm sem of triplicate wells unless otherwise indicated. ${ }^{*} p<0.05 ;{ }^{* *} p<0.01 ;{ }^{* * *} p<0.001$; ${ }^{* * * *} p<0.0001 ;$ ANOVA ELOVL5 knockdown versus control.

Figure 5: Proliferative effects of ELOVL5 knockdown are rescued by cis-vaccenic acid.

(A) Schematic pathway of monounsaturated fatty acid (MUFA) and polyunsaturated fatty acid (PUFA) synthesis, showing elongation and desaturation steps of fatty acids regulated by ELOVL5 that result in production of arachidonic acid (AA), docosahexaenoic acid (DHA) and cis-vaccenic acid (cVA). (B-C) LNCaP cell viability rescue experiments for ELOVL5 knockdown-related changes in response to arachidonic acid (AA; C20:4;omega6), docosahexaenoic acid (DHA; C22:6;omega-3) and cis-vaccenic acid (cVA; C18:1;omega-7) products of ELOVL5 $(5-10 \mu M)$. Data is representative of at least 3 
independent experiments and presented as mean \pm sem of triplicate wells. ${ }^{*} p<0.05$; ${ }^{* *} p<0.01 ;{ }^{* * *} p<0.001 ;{ }^{* * * *} p<0.0001$; ANOVA ELOVL5 knockdown versus control.

Figure 6: ELOVL5 knockdown alters mitochondrial morphology, respiration and reactive oxygen species production. (A) Mitochondrial phenotype changes related to ELOVL5 knockdown in LNCaP cells revealed by electron microscopy, with significant increases in mitochondrial size and length detected. ${ }^{*} p<0.0001$ T-test siELOVL5 versus siCTRL. (B) Seahorse analysis of mitochondrial respiration in ELOVL5-depleted LNCaP cells (top panel) and ELOVL5-overexpressing LNCaP cells (lower panel). Basal and maximal respiration, ATP production, and mitochondrial spare capacity are shown and compared to BSA control. Data is representative of 3 independent experiments and presented as mean \pm sem of triplicate wells. ${ }^{*} p<0.05 ;{ }^{* *} p<0.01 ;{ }^{* * *} p<0.001 ;{ }^{* * *} p<0.0001$; ANOVA ELOVL5 knockdown versus control, ns=not significant. (C) Reactive oxygen species (ROS) production induced by ELOVL5 knockdown, and rescue using antioxidant trolox $(100 \mu \mathrm{M})$ by flow cytometric measurement of CellROX ${ }^{\mathrm{TM}}$ Deep Red Reagent. (D) LNCaP cell viability rescue experiments for ELOVL5 knockdown-related changes in response to $100 \mu \mathrm{M}$ trolox. (E) ROS produced by ELOVL5 knockdown in LNCaP cells, and rescue in the presence of MUFA cVA $(10 \mu \mathrm{M})$ by flow cytometric measurement of CellROX ${ }^{\mathrm{TM}}$ Deep Red Reagent. (F) ROS inhibition upon ELOVL5 overexpression in LNCaP cells. All data is representative of at least 3 independent experiments and presented as mean \pm SEM of triplicate wells. ${ }^{*} p<0.05 ;{ }^{* *} p<0.01 ;{ }^{* * *} p<0.001 ;{ }^{* * *} p<0.0001$; ANOVA ELOVL5 knockdown versus control, ns=not significant.

Figure 7: ELOVL5 knockdown inhibits cell migration, prostate tumor growth and metastasis. (A-B) LNCaP and 22Rv1 prostate cancer cell lines subjected to siRNAmediated ELOVL5 knockdown were analysed for cell migration through Matrigel. Data are representative of at least 2 independent experiments and presented as mean \pm SEM of 
triplicate wells. ${ }^{*} p<0.05,{ }^{* * *} p<0.001$ ANOVA, siELOVL5\#1/\#2 versus siCTRL. (C) LNCaP cells with stable overexpression of ELOVL5 were analysed for cell migration through Matrigel. Data are representative of 2 independent experiments and presented as mean \pm SEM of triplicate wells. ${ }^{* *} p<0.01$ ANOVA, shELOVL5- versus shCTRL. (D) 22Rv1 migration rescue experiments for ELOVL5 knockdown-related changes in response to 10 $\mu \mathrm{M} \mathrm{cVA}$. Data are representative of 3 independent experiments and presented as mean \pm SEM of triplicate wells. ${ }^{*} p<0.05$ ANOVA, cVA versus vehicle control. (E) LNCaP cells constitutively expressing shRNA directed to $\operatorname{ELOVL5}(n=7)$ or control $(n=7)$ were analysed for orthotopic LNCaP tumor growth in mice. Data are presented as mean \pm SEM. ${ }^{*} p<0.05$ T-test, shELOVL5- versus shCTRL. (F) Bioluminescent images of metastatic spread to the lungs of all shCTRL $(n=7)$ and shELOVL5- $(n=7)$ mice from (E). Representative H\&E staining and AR immunostaining of shCTRL mouse lung boxed in red, showing histological evidence of metastatic human LNCaP cells in the mouse lung. Metastasis of orthotopic tumors to the lungs presented as lung luminescence normalised to primary tumor weight. 
Figure 1

A

$\mathbf{B}_{\text {Total lipid abundance }}$

C Abundance per lipid class

D

Prostate cancer cells
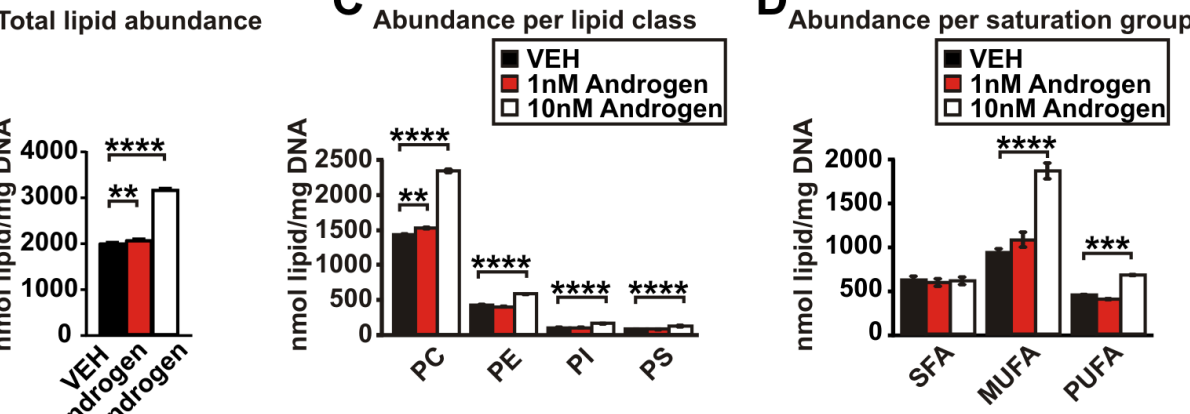

mass spectrometry

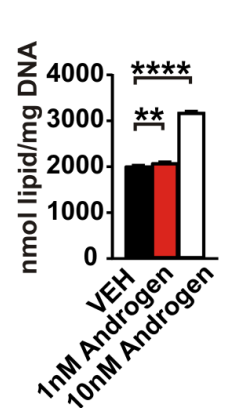

lipidomics

E

Vehicle Androgen

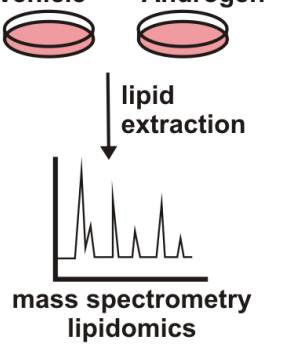

Phospholipid abundance in androgen or anti-androgen treated prostate cancer cells

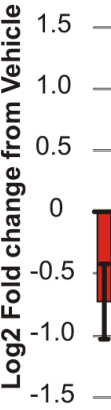

$\square$ 1nM Androgen

$\square 1 \mu \mathrm{M}$ Enzalutamide

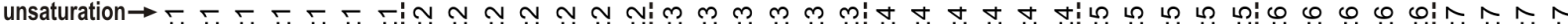

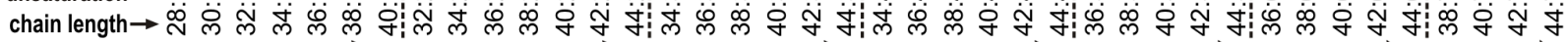

MUFA elongation

$\mathbf{F}$

Prostate tumor

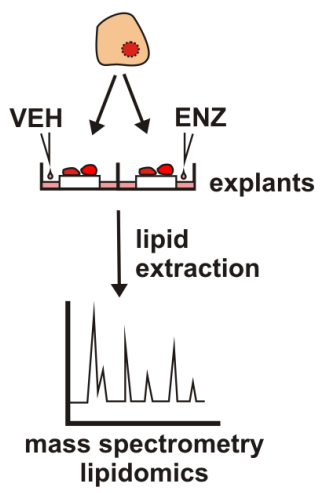

H Influence of androgens on phospholipid structure

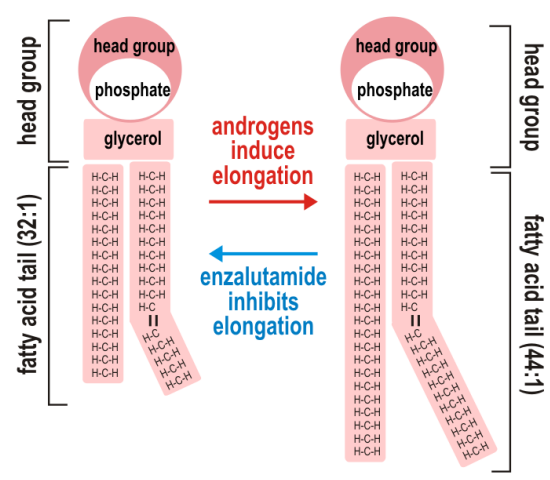

G Phospholipid abundance in antagonist treated patient-derived prostate cancer explants

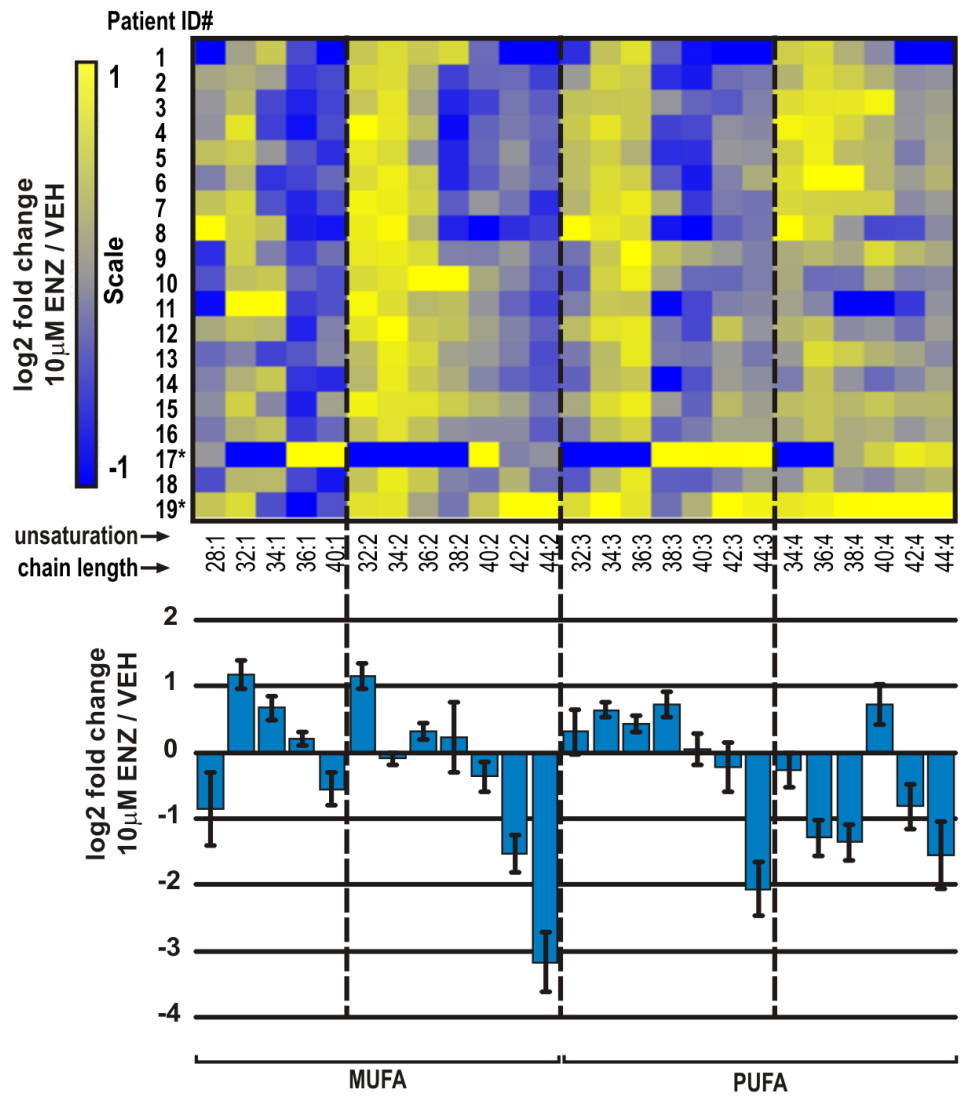


Figure 2
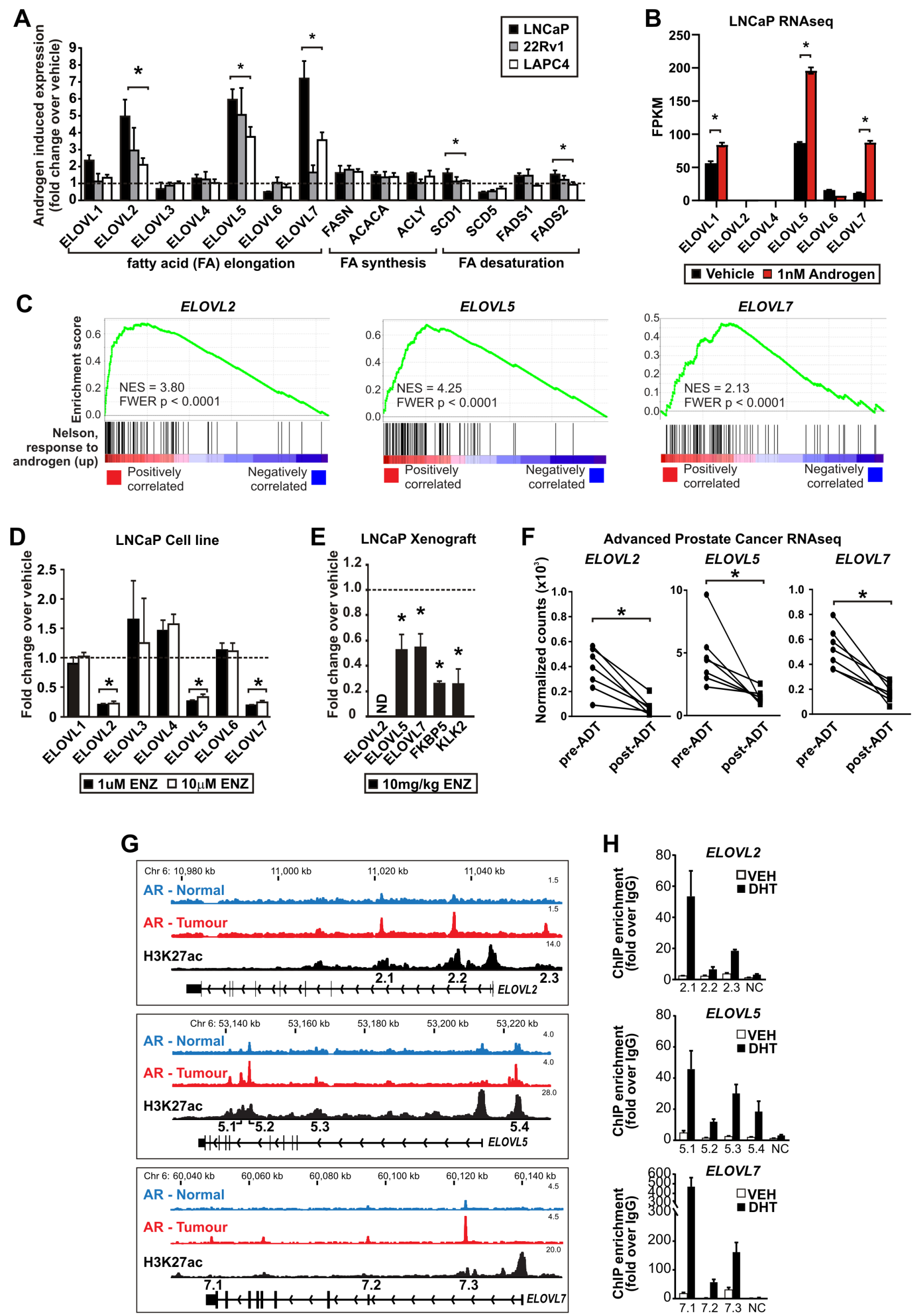
Figure 3

A ELOVL RNA B Phosphatidylcholine (PC) abundance in androgen or siELOVL treated LNCaP cells
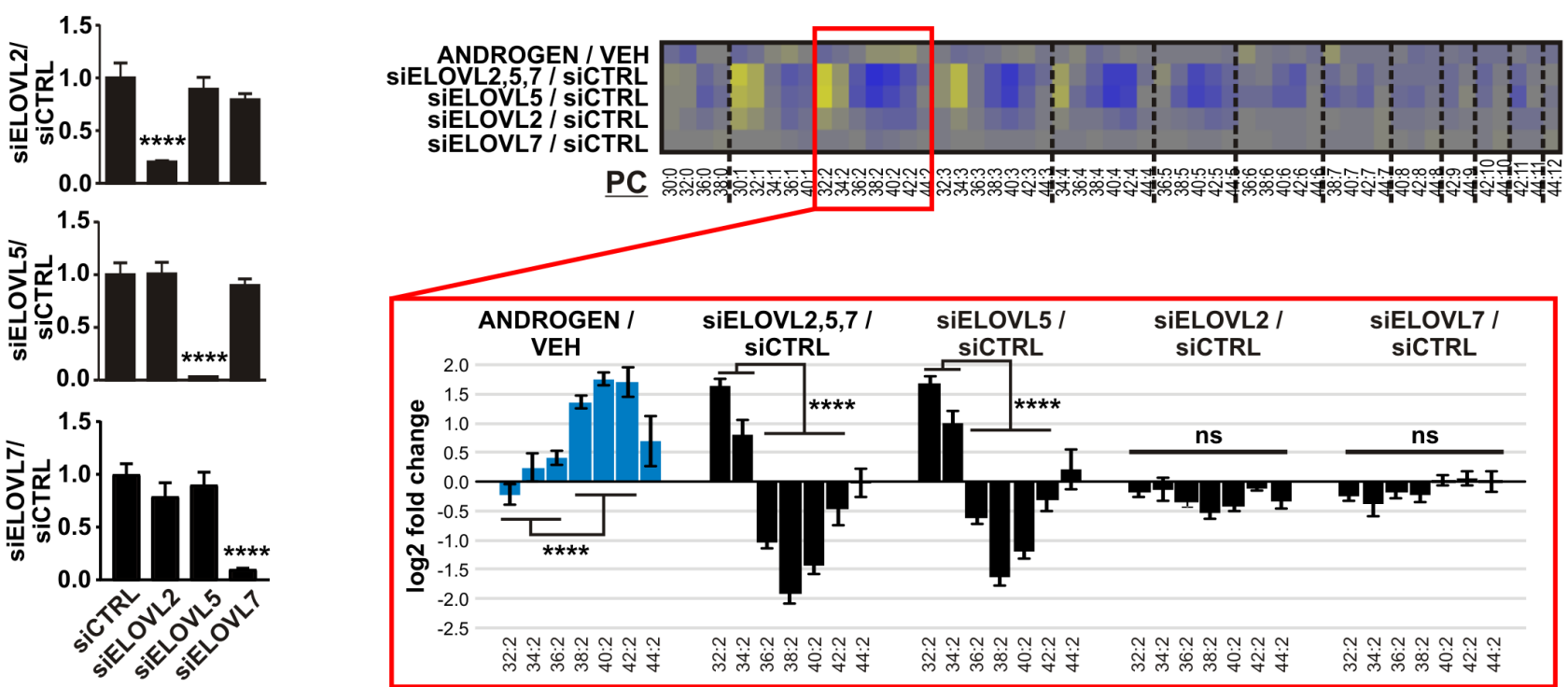

\section{C}

Primary Prostate Cancer (TCGA)

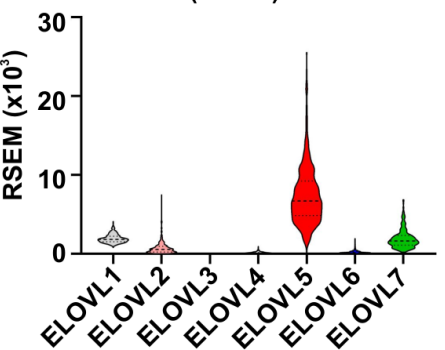

D Metastatic CRPC

(SU2C)

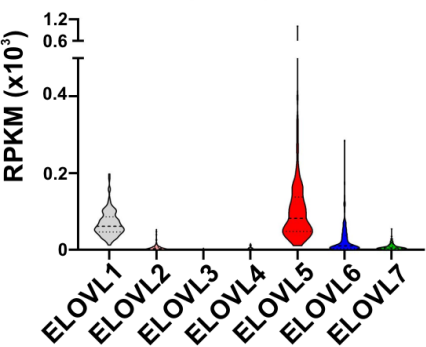

H basal cytokeratin luminal cytokeratin

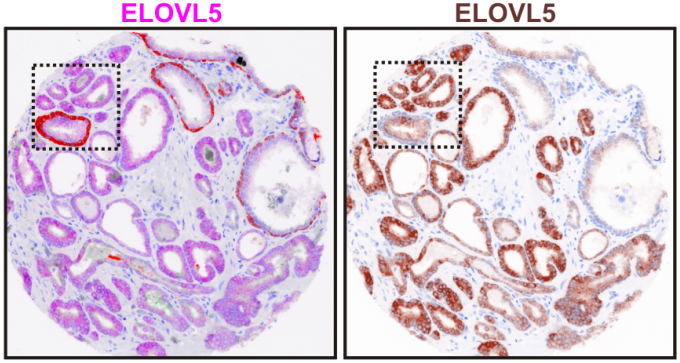

E ELOVL5 RNA

F ELOVL5 RNA

$\mathbf{G}$

ELOVL5
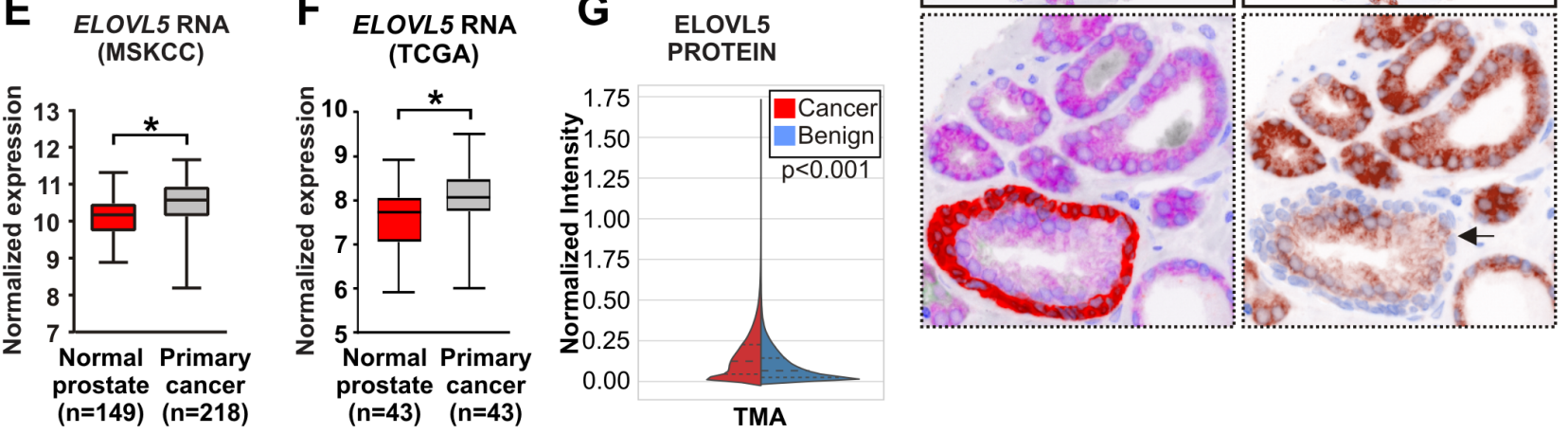

TMA 
Figure 4
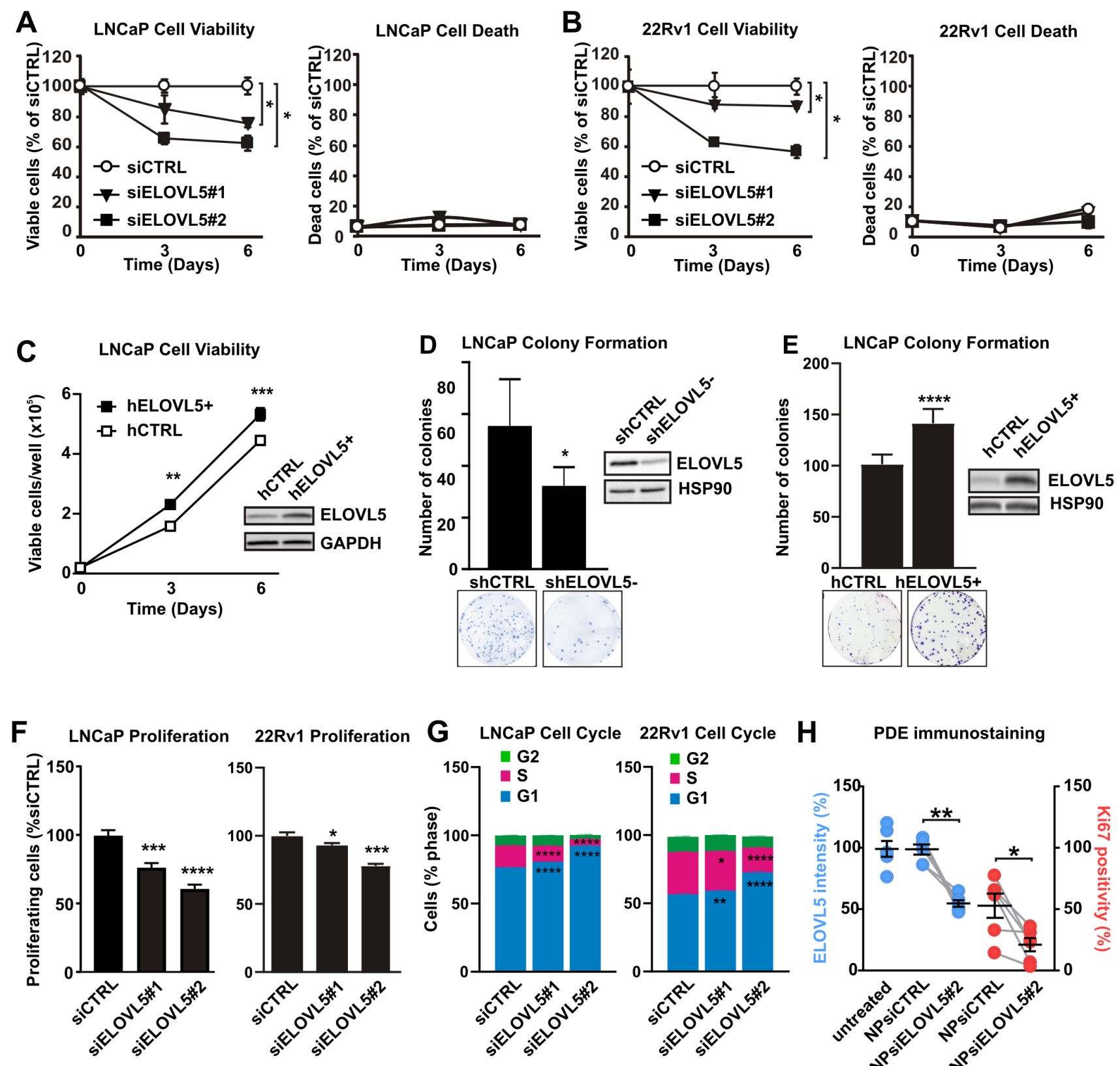

H PDE immunostaining
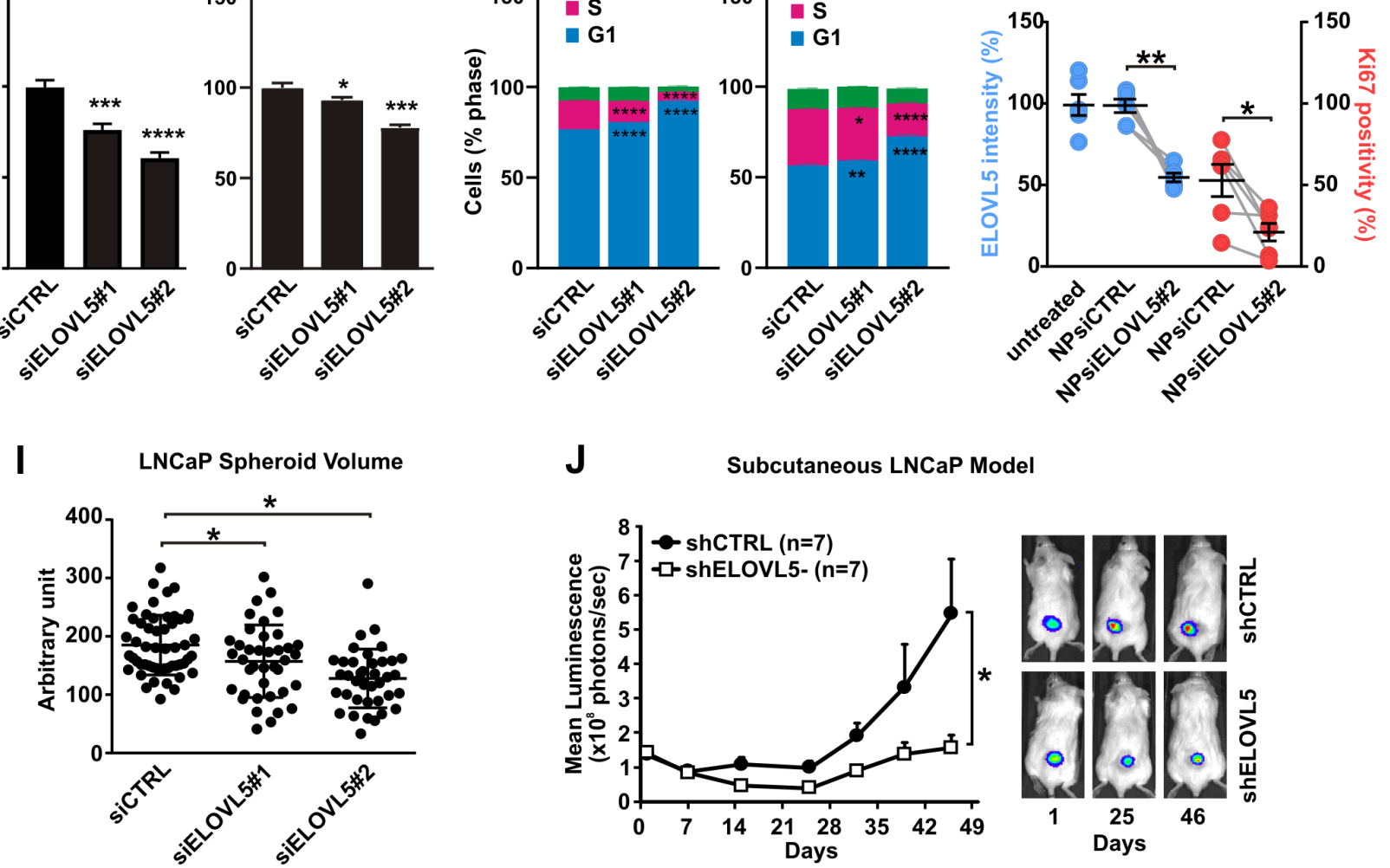

J

Subcutaneous LNCaP Model

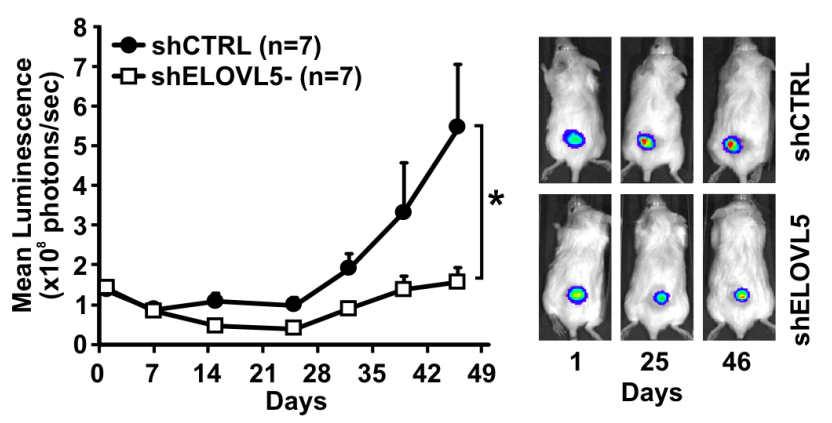




\section{Figure 5}

A

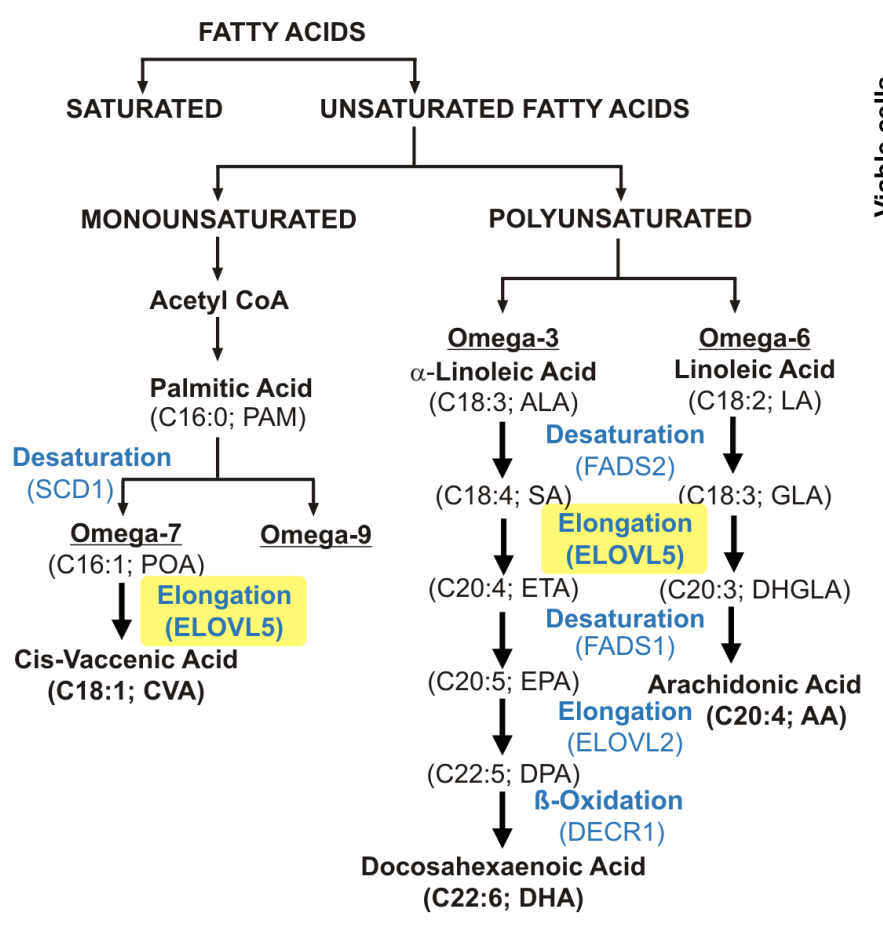

B

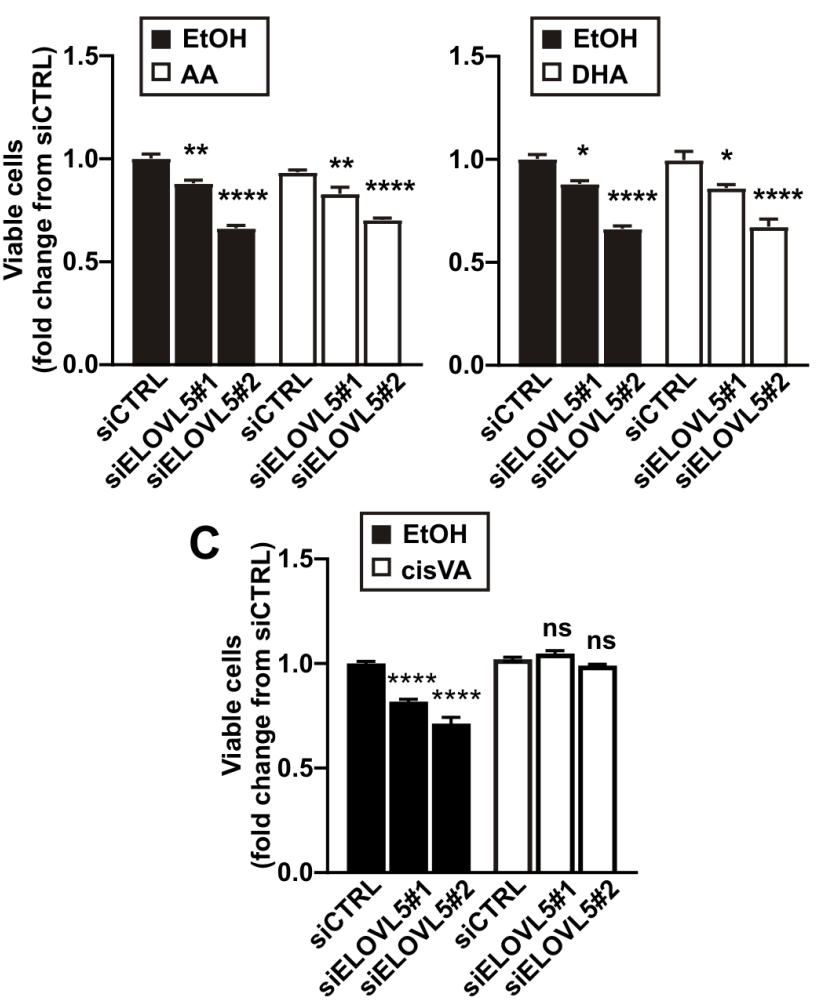


Figure 6

A
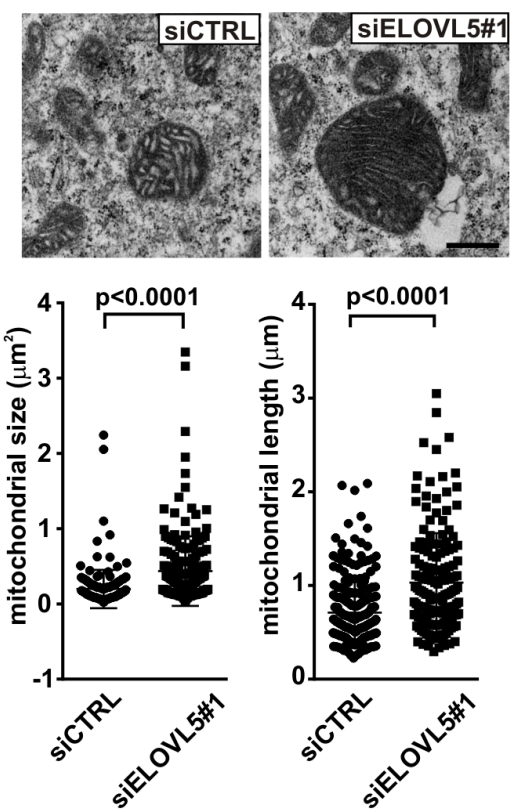

B
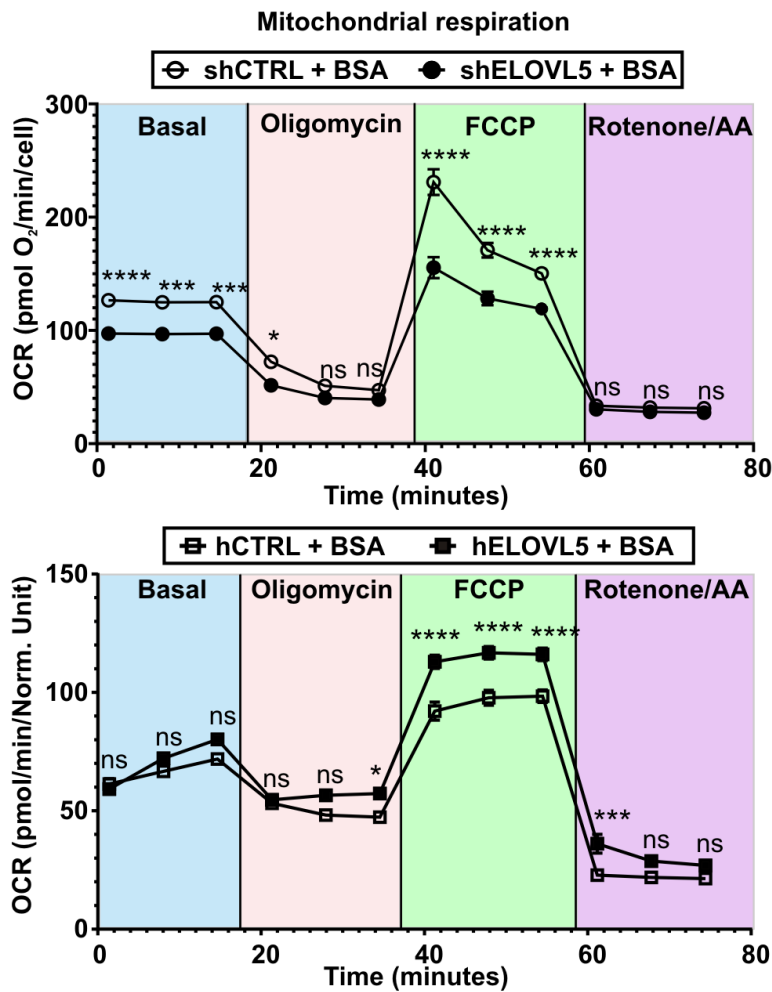

E

LNCaP viability

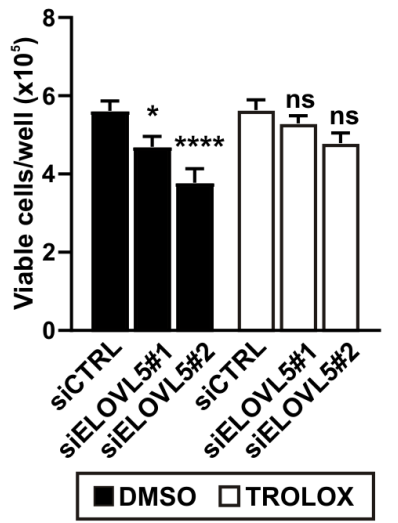

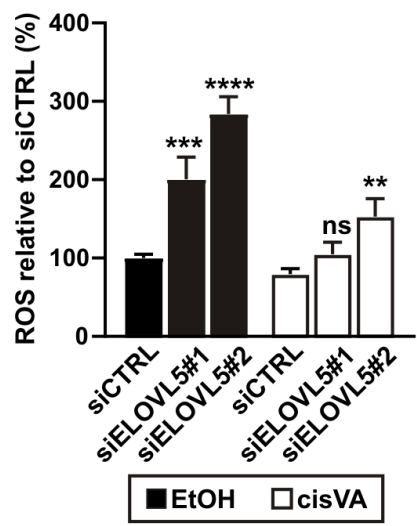

F

Oxidative Stress

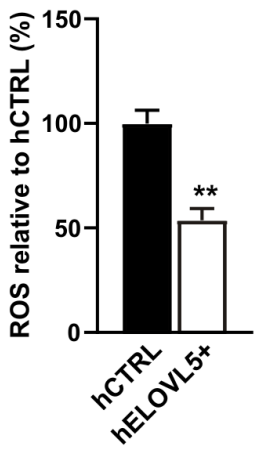


Figure 7

A

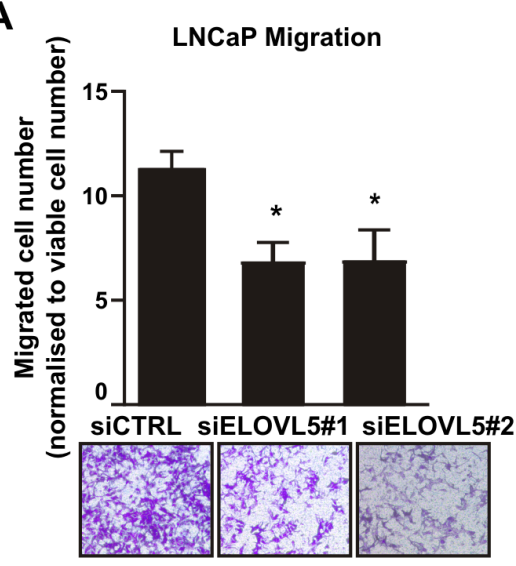

D

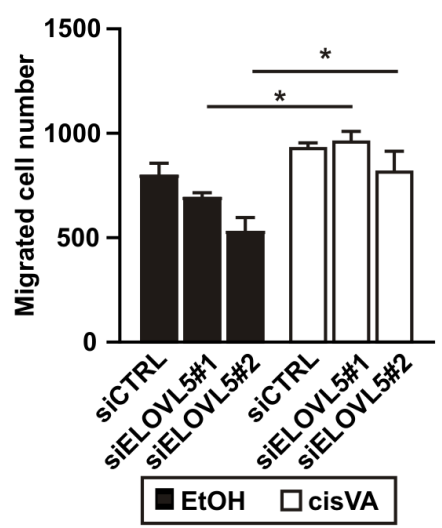

F

Orthotopic LNCaP Model - Lung luminescence

ShCTRL

metastasis in $4 / 7$ mice
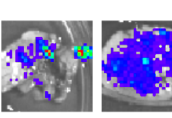

ShELOVL5 metastasis in $0 / 7$ mice
B

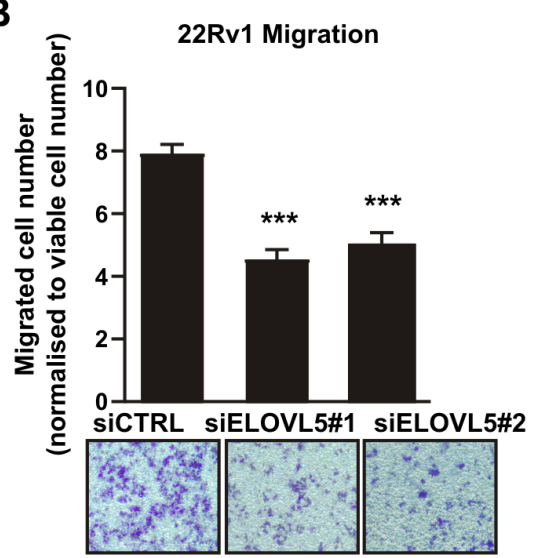

C LNCaP Migration

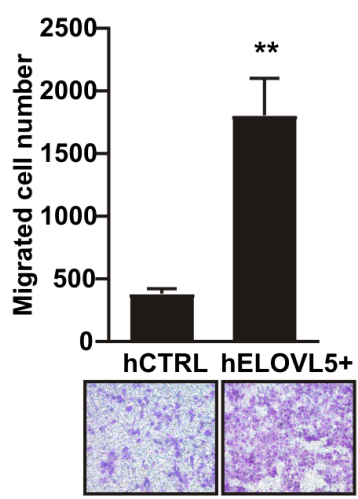

E

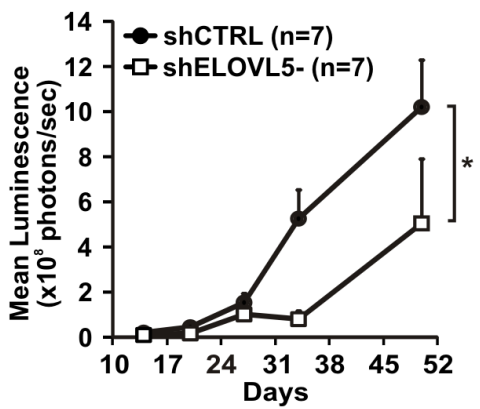

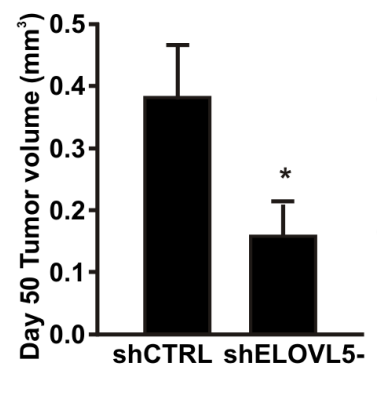

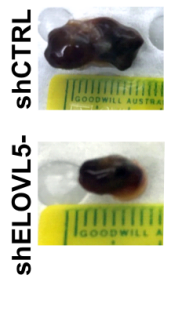

$\frac{\alpha}{4}$
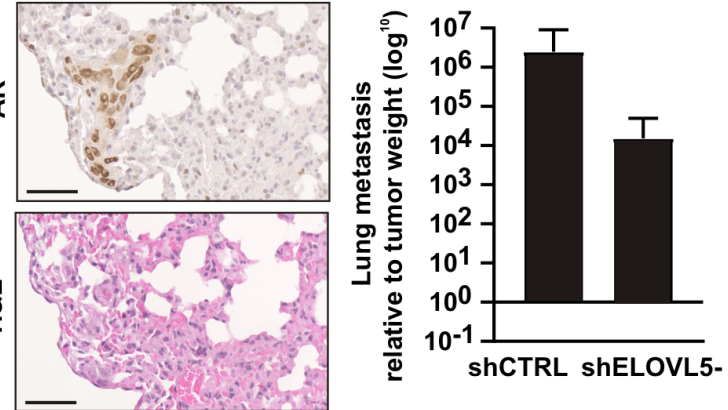